Man wird im allgemeinen das Berechnungsverfahren für den Mindestwert des Vorkontaktwiderstandes dadurch etwas vereinfachen können, daß man für den Wert $\omega \tau$ einen mittleren Betrag, etwa $\omega \tau=90^{\circ}$, einsetzt. Mit diesem Vorschlage erhalten wir

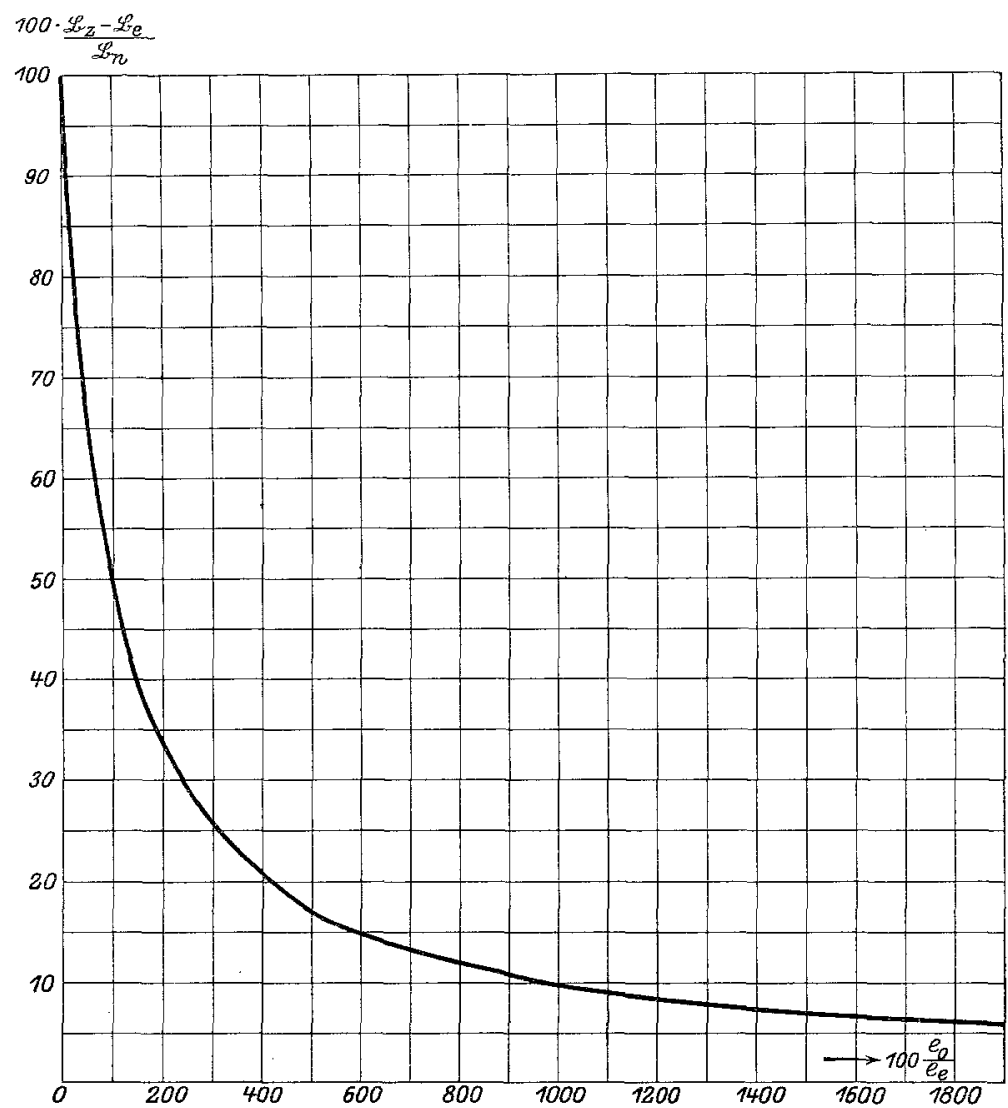

Fig. 24.

Fig. 24, aus der man nun unmittelbar zu dem gegebenen Werte Ioo. $\left(\frac{\mathfrak{B}_{\mathrm{z}}-\mathfrak{B}_{\mathrm{e}}}{\mathfrak{B}_{\mathrm{n}}}\right)$ den gesuchten Wert $\frac{\mathrm{e}_{0}}{\mathrm{e}_{\mathrm{e}}}$ abgreifen und $\mathrm{R}$ berechnen kann.

\title{
Die doppelt verkettete Streuung beim Zweiphasenmotor und beim Dreiphasenstator mit Zweiphasenrotor.
}

\author{
Von
}

\section{Heinrich Meyer-Wülfing.}

\section{Die doppelt verkettete Streuung bei $Z$ weiphasenmotoren.}

1. Allgemeine Übersicht. Im folgenden habe ich, einer Anregung des Herrn Prof. Dr. Simons folgend, es unternommen, gestützt auf die von Rogowski und Simons ${ }^{1}$ ) gegebenen Grundlagen, die doppelt verkettete Streuung des Zweiphasenmotors und des

:) Rogowski, Dissertation; Rogowski und Simons ETZ. I9o8, Heft 22 und 23; ETZ. I909. Heft Io, I I und 52; ETZ. Igro, Heft 5I und 52. Siehe auch: Kittler-Petersen, Allgemeine Elektiotechnik, III. Bd., S. 82 u. f. 
Dreiphasenstators mit $Z$ weiphasenrotor $z u$ untersuchen. Ich setze einen $Z$ weiphasenmotor voraus, der im Stator und Rotor vollkommen symmetrisch gebaut ist, und dem primär eine Spannungswelle von sinusförmiger Gestalt aufgedrückt wird.

Für die Berechnung der Streuung dieses Motors ergeben sich nun drei Wege:

I. Man legt bei geöfnetem, stillstehendem Rotor den Stator an die (normale) Spannung $E_{1}$ und mißt gleichzeitig die im Rotor induzierte Spannung $E_{2}$; ist $\mathrm{p}=\mathrm{N}_{1} / \mathrm{N}_{2}$ das Verhältnis der Windungszahlen pro Phase primär und sekundär, dann bezeichnet man die Differenz

$$
\mathrm{E}_{\mathrm{s}_{\mathrm{i}}}=\mathrm{E}_{1}-\mathrm{p} \mathrm{E}_{2}
$$

als primäre Streuspannung und nennt den Quotienten

$$
\tau_{1}=\frac{\mathrm{E}_{\mathrm{s}_{1}}}{\mathrm{p \textrm {E } _ { 2 }}}
$$

den ,primären, einfachen Streukoeffizienten“"

Will man den entsprechenden sekundären Streukoeffizienten expërimentell ermitteln, so muß man umgekehrt dem Rotor einen Strom zuführen, die an den Schleifringen erforderliche Spannung und gleichzeitig die im Stator induzierte Spannung messen. Es ergibt sich dann:

$$
\tau_{2}=\frac{p E_{\mathrm{s}_{2}}}{\mathrm{E}_{1}}
$$

wo

$$
\mathrm{E}_{\mathrm{s}_{2}}=\mathrm{E}_{2}-\frac{\mathrm{I}}{\mathrm{p}} \mathrm{E}_{1}
$$

Aus den beiden Koeffizienten bilden wir die Kombination

$$
\sigma=\tau_{1}+\tau_{2}+\tau_{1} \cdot \tau_{2}
$$

die letzten Endes allein interessiert.

Diese Methode stammt von Blondelli). Daß sie völlig übereinstimmende Werte mit dem idealen He ylandschen Kreisdiagramm gibt, ist von Rogowski²) und Simons ${ }^{2}$ ) nachgewiesen worden.

Formelmäßig kann man die eben gegebene Vorschrift wie folgt berücksichtigen:

Unter Verwendung der üblichen Bezeichnungsweise kennzeichnen wir die primären Phasen mit a und $\mathrm{b}$, die sekundären mit $\mathrm{x}$ und $\mathrm{y}$, Momentanwerte mit kleinen Buchstaben unter Beifügung eines t, Amplituden mit großen Buchstaben, den Widerstand einer primären Phase mit W, ihre Eigeninduktivität mit L, den. Widerstand einer sekundären Phase mit w, ihre Eigeninduktivität mit 1, gegenseitige Induktivitäten mit M.

Bei ideellem Leerlauf müssen sich naturgemäß die Gleichungen des einfachen Wechselstromkreises mit Widerstand und Selbstinduktion ergeben, da das Vorhandensein des stillstehenden, geöffneten Rotors für die elektrischen Verhältnisse des Stators ohne Belang ist.

Aus den Gleichungen:

$$
\begin{aligned}
& e_{a t}=E \cdot \sin \omega t \quad \text { und } \quad e_{b t}=E \cdot \cos \omega t \\
& e_{x t}=M_{a x} \cdot \frac{d i_{a}{ }^{0}}{d t}+M_{b x} \cdot \frac{{d i_{b}{ }^{0}}_{d t}}{}
\end{aligned}
$$

und

$$
e_{y}=M_{a y} \cdot \frac{d i_{a}{ }^{0}}{d t}+M_{b y} \cdot \frac{d i_{b}{ }^{0}}{d t}
$$

1) 'Blondel, L'éclairage électrique 189.5 , Seite 364 .

2) Rogowski, E. u. M. I9I2, Heft 9; Rogowski und Simons ETZ. I909, Heft Io und II. 
folgen ohne weiteres, da aus Symmetriegründen für jede Stellung noch die Beziehungen gelten:

die Resultate:

$$
\begin{aligned}
& \mathrm{M}_{\mathrm{ax}}=\mathrm{M}_{\mathrm{by}}=\mathrm{M}_{1} \\
& \mathrm{M}_{\mathrm{ay}}=\mathrm{M}_{\mathrm{bx}}=\mathrm{M}_{2}
\end{aligned}
$$

$$
\begin{aligned}
\mathrm{E}_{1} & =\mathrm{J}_{0} \cdot \sqrt{\mathrm{W}^{2}+\omega^{2} \mathrm{~L}^{2}} \\
\operatorname{tg} \varphi & =\frac{\omega \mathrm{L}}{\mathrm{W}} \\
\mathrm{E}_{2} & =\mathrm{J}_{0} \cdot \sqrt{\mathrm{M}_{1}^{2}+\mathrm{M}_{2}^{2}} \\
\operatorname{tg} \psi & =\frac{\mathrm{M}_{1}}{\mathrm{M}_{2}},
\end{aligned}
$$

wo $\varphi$ der Phasenverschiebung zwischen Spannung und Strom primär, $\psi$ der Phasenverschiebung zwischen Spannung sekundär und Strom primär Rechnung trägt.

Für den vorliegenden Fall lauten die Resultate:

$$
\begin{gathered}
\tau_{1}=\frac{\mathrm{L}-\mathrm{p} \cdot \sqrt{\mathrm{M}_{1}{ }^{2}+\mathrm{M}_{2}{ }^{2}}}{\mathrm{p} \cdot \sqrt{\mathrm{M}_{1}{ }^{2}+\mathrm{M}_{2}{ }^{2}}} \\
\tau_{2}=\frac{\mathrm{p} \cdot 1-\sqrt{\mathrm{M}_{1}{ }^{2}+\mathrm{M}_{2}{ }^{2}}}{\sqrt{\mathrm{M}_{1}{ }^{2}+\mathrm{M}_{2}{ }^{2}}} \\
\sigma=\frac{\mathrm{L} \cdot 1-\left\{\mathrm{M}_{1}{ }^{2}+\mathrm{M}_{2}{ }^{2}\right\}}{\mathrm{M}_{1}{ }^{2}+\mathrm{M}_{2}{ }^{2}} .
\end{gathered}
$$

In der Praxis rechnet man im allgemeinen nicht mit den hier benutzten Induktivitäten, sondern mit Feldern und Spulenfaktoren. Mit deren Hilfe hat man die obigen Gleichungen folgendermaßen zu schreiben: Wir führen die Kraftlinien $p$ für eine Amperewindung (primär) ein; dann ist die primäre Spannung:

oder

$$
E_{1}=-\mathrm{L} \cdot \frac{\mathrm{di}_{1}}{\mathrm{dt}}=-\mathrm{N}_{1}^{2} \cdot \mathrm{f} \cdot \varphi \frac{\mathrm{di}}{\mathrm{dt}} \cdot \mathrm{IO}^{-8} \text { Volt }
$$

$$
\mathrm{L}=\mathrm{N}_{1}^{2} \cdot \mathrm{f}_{\mathrm{\rho}} \mathrm{IO}^{-8} \ldots \ldots . . . . . \text { Henry. }
$$

Durch Einsetzen des Wertes für $\varrho$ finden wir dann endlich:

$$
\begin{aligned}
L & =N_{1}{ }^{2}\left\{f_{1} \varphi_{1}+f_{1 s} \varphi_{1 s}\right\} \cdot I^{-8} \ldots \ldots . . . \text { Henry } \\
I & =N_{2}{ }^{2}\left\{f_{2} \varphi_{2}+f_{2 s} \varphi_{2 s}\right\} \cdot I^{-8} \ldots \ldots . \text { Henry } \\
M & =N_{1} N_{2} f_{21} \varphi_{2} \cdot I O^{-8}=N_{1} N_{2} f_{12} \varphi_{1} I^{-8} \text { Henry. }
\end{aligned}
$$

Damit wir also die Koeffizienten $L, 1, M_{1}$ und $M_{2}$ einfach wie Spulenfaktoren ermitteln können, ist die Kenntnis der fiktiven Felder nötig. Ferner muß die Gleichung erfüllt sein

$$
\mathrm{f}_{12} \varphi_{1}=\mathrm{f}_{21} \varphi_{2} .
$$

Dies trifft aber, wie leicht einzusehen, nur dann zu, wenn die Amperewindungszahlen primär und sekundär einander gleich sind, z. B. für gleiche Stromstärken bei dem Übersetzungsverhältnis $\mathrm{N}_{1} / \mathrm{N}_{2}=$ I. Bei gleichen Windungszahlen und gleicher Stromstärke ergeben sich die Koeffizienten der Induktion einfach als die für die betrachtete Stellung vorhandenen Kraftlinienverkettungen.

2. Bei einem zweiten, von He yland angegebenen Wege hat man einen ideellen Motor vorauszusetzen $\{\mathrm{W}=0, \mathrm{~W}=0 ; \mathrm{W}=$ Widerstand im Stator, $\mathrm{w}=$ Widerstand im Rotor $\}$ 
und für eine und dieselbe primäre Spannung $\mathrm{E}_{1}$ den Leerlaufsstrom $\mathrm{J}_{0}$ und den Kurzschlußstrom $\mathrm{J}_{\mathrm{k}}$ zu berechnen und endlich das Verhältnis

$$
\sigma=\frac{\mathrm{J}_{0}}{\mathrm{~J}_{\mathrm{k}}-\mathrm{J}_{0}}
$$

$\left\{\right.$ häufig noch vereinfacht $\left.z u \sigma=\frac{J_{0}}{J_{\mathrm{k}}}\right\}$ zu bilden. Nun ist, wenn wir mit $\chi$ die Phasenverschiebung der primären Ströme untereinander bezeichnen:

$$
\begin{aligned}
& \mathrm{E}_{1} \cdot \sin (\omega t+\varphi)=\mathrm{J}_{\mathrm{k}}^{\mathrm{a}} \cdot \omega \mathrm{L} \cos \omega \mathrm{t}+\mathrm{M}_{1} \frac{\mathrm{di}_{\mathrm{x}}}{\mathrm{dt}}+\mathrm{M}_{2} \frac{\mathrm{di}_{\mathrm{yt}}}{\mathrm{dt}} \\
& \mathrm{E}_{1} \cdot \cos (\omega \mathrm{t}+\varphi)=\mathrm{J}_{\mathrm{k}}^{\mathrm{b}} \cdot \omega \mathrm{L} \cos (\omega \mathrm{t}+\chi)+\mathrm{M}_{2} \frac{\mathrm{di}_{\mathrm{xt}}}{\mathrm{dt}}+\mathrm{M}_{1} \frac{\mathrm{di}_{\mathrm{yt}}}{\mathrm{dt}} \\
& \mathrm{e}_{\mathrm{xt}}=0=1 \frac{\mathrm{di} \mathrm{xt}}{\mathrm{dt}}+\mathrm{J}_{\mathrm{k}}^{\mathrm{a}} \omega \mathrm{M}_{1} \cos \omega \mathrm{t}+\mathrm{J}_{\mathrm{k}}^{\mathrm{b}} \omega \mathrm{M}_{2} \cos (\omega \mathrm{t}+\chi) \\
& \mathrm{e}_{\mathrm{y} t}=0=1 \frac{\mathrm{di} \mathrm{t}}{\mathrm{dt}}+\mathrm{J}_{\mathrm{k}}^{\mathrm{a}} \omega \mathrm{M}_{2} \cos \omega \mathrm{t}+\mathrm{J}_{\mathrm{k}}^{\mathrm{b}} \omega \mathrm{M}_{1} \cos (\omega \mathrm{t}+\chi)
\end{aligned}
$$

Nach den letzten beiden Gleichungen können $i_{x t}$ und $i_{y t}$ ebenfalls nur einfache Sinusfunktionen sein, wir setzen demzufolge:

und

$$
\mathrm{i}_{\mathrm{xt}}=\mathrm{J}_{\mathrm{x}} \cdot \sin (\omega \mathrm{t}+\xi)
$$

$$
i_{y t}=J_{y} \cdot \sin (\omega t+\zeta)
$$

und erhalten zur Berechnung der acht Unbekannten

$$
\varphi, \mathrm{J}_{\mathrm{k}}^{\mathrm{a}}, \mathrm{J}_{\mathrm{k}}^{\mathrm{b}}, \chi, \mathrm{J}_{\mathrm{x}}, \mathrm{J}_{\mathrm{y}}, \xi \text { und } \zeta
$$

die folgenden acht Gleichungen

$$
\begin{aligned}
\mathrm{E}_{1} \cos \varphi & =-\mathrm{J}_{\mathrm{x}} \omega \mathrm{M}_{1} \sin \xi-\mathrm{J}_{\mathrm{y}} \omega \mathrm{M}_{2} \sin \zeta \\
\mathrm{E}_{1} \sin \varphi & =\mathrm{J}_{\mathrm{k}}^{\mathrm{a}} \cdot \omega \mathrm{L}+\mathrm{J}_{\mathrm{x}} \omega \mathrm{M}_{1} \cos \xi+\mathrm{J}_{\mathrm{y}} \omega \mathrm{M}_{2} \cos \zeta \\
\mathrm{E}_{1} \cos \varphi & =\mathrm{J}_{\mathrm{k}}^{\mathrm{b}} \omega \mathrm{L} \cos \chi+\mathrm{J}_{\mathrm{x}} \omega \mathrm{M}_{2} \cos \xi+\mathrm{J}_{\mathrm{y}} \omega \mathrm{M}_{1} \cos \zeta \\
\mathrm{E}_{1} \sin \varphi & =\mathrm{J}_{\mathrm{k}}^{\mathrm{b}} \omega \mathrm{L} \sin \chi+\mathrm{J}_{\mathrm{x}} \omega \mathrm{M}_{2} \sin \xi+\mathrm{J}_{\mathrm{y}} \omega \mathrm{M}_{1} \sin \zeta \\
0 & =-\mathrm{J}_{\mathrm{x}} \omega 1 \sin \xi-\mathrm{J}_{\mathrm{k}}^{\mathrm{b}} \omega \mathrm{M}_{2} \sin \chi \\
o & =\mathrm{J}_{\mathrm{x}} \omega 1 \cos \xi+\mathrm{J}_{\mathrm{k}}^{\mathrm{a}} \omega \mathrm{M}_{1}+\mathrm{J}_{\mathrm{k}}^{\mathrm{b}} \omega \mathrm{M}_{2} \cos \chi \\
o & =-\mathrm{J}_{\mathrm{y}} \omega 1 \sin \zeta-\mathrm{J}_{\mathrm{k}}^{\mathrm{b}} \omega \mathrm{M}_{1} \sin \chi \\
o & =\mathrm{J}_{\mathrm{y}} \omega 1 \cos \zeta+\mathrm{J}_{\mathrm{k}}^{\mathrm{a}} \omega \mathrm{M}_{2}+\mathrm{J}_{\mathrm{k}}^{\mathrm{b}} \omega \mathrm{M}_{1} \cos \chi .
\end{aligned}
$$

Als wesentliche Erleichterung nehmen wir an Stelle der primären Spannungswelle die primäre Stromwelle an; es sei

und

$$
\dot{\mathrm{J}}_{\mathrm{k}}^{\mathrm{a}}=\mathrm{J}_{\mathrm{k}}^{\mathrm{b}}=\mathrm{J}_{1 \mathrm{k}}
$$

$$
\chi=\frac{\pi}{2}
$$

unsere früheren Gleichungen lauten jetzt
I) $\mathrm{E}_{1} \cos \varphi=-\mathrm{J}_{\mathrm{x}} \omega \mathrm{M}_{1} \sin \xi-\mathrm{J}_{\mathrm{y}} \omega \mathrm{M}_{2} \sin \zeta$
2) $\mathrm{E}_{1} \sin \varphi=\mathrm{J}_{1 \mathrm{k}} \omega \mathrm{L}+\mathrm{J}_{\mathrm{x}} \omega \mathrm{M}_{1} \cos \xi+\mathrm{J}_{\mathrm{y}} \omega \mathrm{M}_{2} \cos \zeta$
3) $\mathrm{E}_{1}^{\prime} \cos \varphi^{\prime}=\mathrm{J}_{\mathrm{x}} \omega \mathrm{M}_{2} \cos \xi+\mathrm{J}_{\mathrm{y}} \omega \mathrm{M}_{1} \cos \zeta$ 


$$
\begin{aligned}
& \text { 4) } \mathrm{E}_{1}{ }^{\prime} \sin \varphi^{\prime}=\mathrm{J}_{1 \mathrm{k}} \omega \mathrm{L}+\mathrm{J}_{\mathrm{x}} \omega \mathrm{M}_{2} \sin \xi+\mathrm{J}_{\mathrm{y}} \omega \mathrm{M}_{1} \sin \zeta \\
& \text { 5) } \quad 0=-\mathrm{J}_{\mathrm{x}} \omega \mathrm{I} \sin \xi-\mathrm{J}_{1 \mathrm{k}} \omega \mathrm{M}_{2} \\
& \text { 6) } \quad 0=\mathrm{J}_{\mathrm{x}} \omega \mathrm{l} \cos \xi+\mathrm{J}_{1 \mathrm{k}} \omega \mathrm{M}_{1} \\
& \text { 7) } 0=-\mathrm{J}_{\mathrm{y}} \omega 1 \sin \zeta-\mathrm{J}_{1 \mathrm{k}} \omega \mathrm{M}_{1} \\
& \text { 8) } \quad 0=J_{\mathrm{y}} \omega 1 \cos \zeta+J_{1 k} \omega M_{2}
\end{aligned}
$$

Gleichung 5 und 8 ergeben ohne weiteres:

$$
\begin{gathered}
\zeta=\xi-\frac{\pi}{2} \\
\mathrm{~J}_{\mathrm{x}}=\mathrm{J}_{\mathrm{y}}=\mathrm{J}_{2 \mathrm{k}} .
\end{gathered}
$$

Aus den Gleichungen $\mathrm{I} \div 4$ findet man:

$$
\begin{aligned}
\mathrm{E}_{1}^{\prime} & =\mathrm{E}_{1} \\
\varphi^{\prime} & =\varphi
\end{aligned}
$$

Es bleiben uns also noch die vier Größen $J_{1 \mathrm{k}}, \varphi, J_{2 \mathrm{k}}$ und $\xi_{3}$, zu deren Bestimmung wir folgende vier Gleichungen benutzen:

$$
\begin{aligned}
\mathrm{E}_{1} \cos \varphi & =-\mathrm{J}_{2 \mathrm{k}} \omega \mathrm{M}_{1} \sin \xi+\mathrm{J}_{2 \mathrm{k}} \omega \mathrm{M}_{2} \cos \xi \\
\mathrm{E}_{1} \sin \varphi & =\mathrm{J}_{1 \mathrm{k}} \omega \mathrm{L}+\mathrm{J}_{2 \mathrm{k}} \omega \mathrm{M}_{1} \cos \xi+\mathrm{J}_{2 \mathrm{k}} \omega \mathrm{M}_{2} \sin \xi \\
o & =-\mathrm{J}_{2 \mathrm{k}} \omega 1 \sin \xi-\mathrm{J}_{1 \mathrm{k}} \omega \mathrm{M}_{2} \\
0 & =\mathrm{J}_{2 \mathrm{k}} \omega \mathrm{l} \cos \xi+\mathrm{J}_{1 \mathrm{k}} \omega \mathrm{M}_{1} .
\end{aligned}
$$

Aus den letzten beiden Gleichungen folgt ohne weiteres

Durch Einführung der Werte

$$
\operatorname{tg} \xi=\frac{\mathrm{M}_{2}}{\mathrm{M}_{1}} \text {. }
$$

$$
\sin \xi=\frac{\mathrm{M}_{2}}{\sqrt{\mathrm{M}_{1}{ }^{2}+\mathrm{M}_{2}{ }^{2}}} \text { und } \cos \xi=\frac{\mathrm{M}_{1}}{\sqrt{\mathrm{M}_{1}{ }^{2}+\mathrm{M}_{2}{ }^{2}}}
$$

in die eben genannten Gleichungen und durch Auflösung nach den Unbekannten erhält man:

$$
\mathrm{J}_{1 \mathrm{k}}=\frac{\mathrm{E}_{1} \cdot 1}{\omega \cdot\left\{\mathrm{L} \cdot \mathrm{l}-\left(\mathrm{M}_{1}{ }^{2}+\mathrm{M}_{2}^{2}\right)\right\}} .
$$

Bilden wir schließlich mit den gefundenen Werten für $J_{0}$ und $J_{k}$ das Verhältnis $\frac{\mathrm{J}_{0}}{\mathrm{~J}_{\mathrm{k}}-\mathrm{J}_{0}}$, so erhalten wir wie oben $(\mathrm{S} .365)$ :

$$
\sigma=\frac{L \cdot 1-\left\{\mathrm{M}_{1}^{2}+\mathrm{M}_{2}^{2}\right\}}{\mathrm{M}_{1}^{2}+\mathrm{M}_{2}^{2}}
$$

3. Es gibt noch einen dritten Weg zur Bestimmung der Streuung; der besteht darin daß man sowohl den Stator als auch den Rotor mit Strom speist. Die Amplitude der Amperewindungszahlen primär und sekundär muß genau übereinstimmen, jedoch die sie erzeugenden Ströme müssen eine Phasenverschiebung gegeneinander haben, die gegeben ist durch den Ausdruck;

$$
\operatorname{tg} \xi=\frac{M_{2}}{M_{1}}
$$

(Dies ist derselbe Winkel, der sich aus den letzten beiden der obigen vier Gleichungen ergeben hat.) Der Beweis ist vollständig von Rogowski, E. und M. I9I2, Seite I97, angegeben und soll hier übergangen werden. 


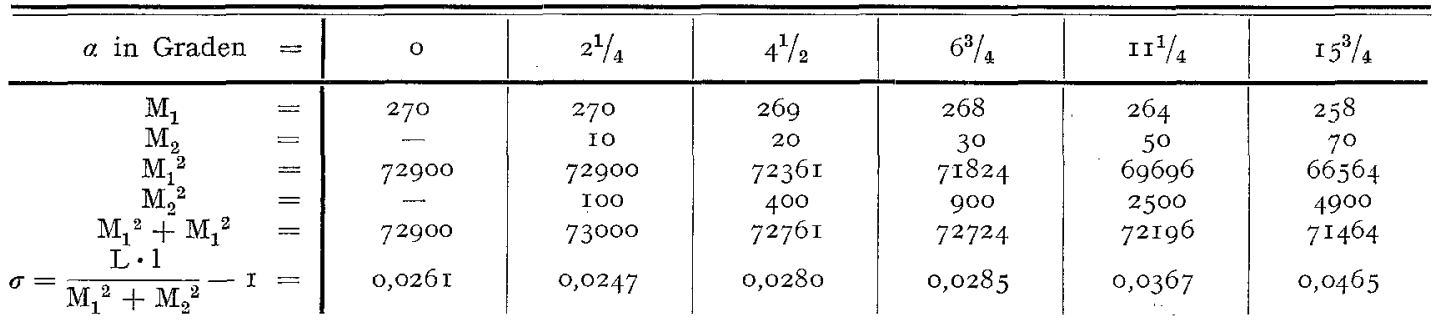

2. Rechnerische Ermittlung der Streukoeffizienten. Nach dem soeben Gesagten können wir leicht die gesuchten Koeffizienten bestimmen. Wir folgen dabei der Methode I. Die fiktiven Felder zeichnen wir nach der Methode von Görges ${ }^{\mathbf{1}}$ ) und ordnen unsere Win-

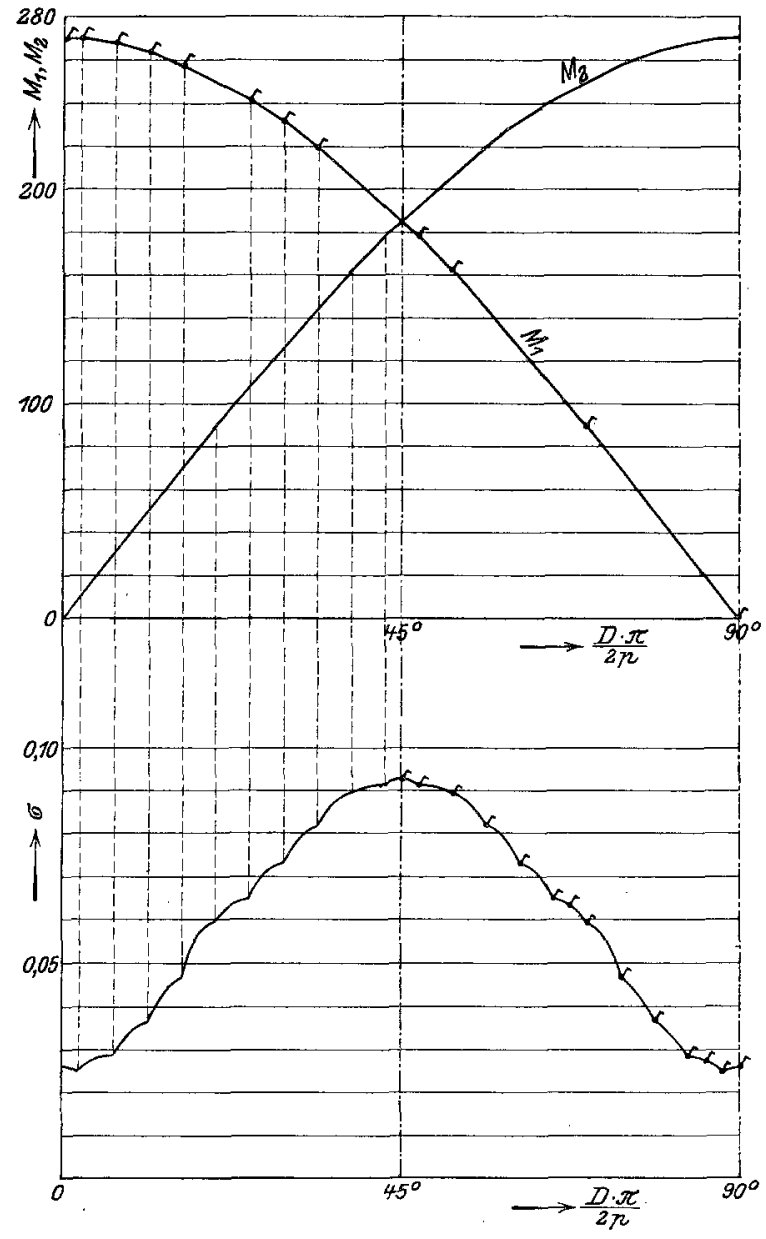

Fig. I. dungen wie folgt an:

Ist die Nutenzahl des Stators pro Pol und Phase gleich n, die entsprechende des Rotors gleich $\mathrm{m}$, dann legen wir in jede Statornut $m$, in jede Rotornut dagegen n Drähte, so daß also beiderseits das Feld durch $m \cdot n$ Drahtwindungen erzeugt wird.

Sodann betrachten wir primär und sekundär nur eine Phase und zwar in dem Moment, in welchem in ihr der Strom seinen Maximalwert hat, die zweite Phase also stromlos ist.

Unter den möglichen Stellungen des Rotors zum Stator gibt es dann ebenfalls, wie beim Drehstrommotor, zwei ausgezeichnete Stellungen: in der einen decken sich die Phasenmitten primär und sekundär - wir wollen dies die, ,erste Hauptstellung nennen - in der anderen liegt die Rotorphase genau zwischen zwei Statorphasen - wir bezeichnen dies als die ,zweite Hauptstellung“.

In den Untersuchungen von Rogowski und Simons ist der Vorschlag gemacht, die Streukoeffizienten alsden arithmetischen Mittelwert aus beiden Hauptstellungen anzugeben, da dieser Wert für fast alle praktischen Bedürfnisse eine hinlänglich genaue Annäherung an den wahren Mittelwert bedeute. Der berechtigte Einwurf Hellmunds, daß dies für kleine und insbesondere für gleiche Nutenzahlen primär und sekundär nicht der Fall ist, hat Rogowski zur exakten Untersuchung der Änderung der Streuung beim Dreiphsaenmotor veranlaßt²).

Bei Annahme treppenförmiger Feldkurven findet man eine Anzahl charakteristischer Punkte, zwischen denen der Verlauf des gegenseitigen Induktionskoeffizienten ein linearer 


\begin{tabular}{|c|c|c|c|c|c|c|c|}
\hline $20^{1} / 4$ & $22^{1} / 2$ & $24^{3 / 4}$ & $29^{1 / 4}$ & $33^{3} / 4$ & $3^{81 / 4}$ & $421 / 2$ & 45 \\
\hline $\begin{array}{r}250 \\
90\end{array}$ & $\begin{array}{r}246 \\
99\end{array}$ & $\begin{array}{l}242 \\
108\end{array}$ & $\begin{array}{l}232 \\
\text { I } 26\end{array}$ & $\begin{array}{l}220 \\
\mathrm{I} 44\end{array}$ & $\begin{array}{l}206 \\
162\end{array}$ & $\begin{array}{l}\text { I92 } \\
\text { I } 78\end{array}$ & $\begin{array}{l}185 \\
185\end{array}$ \\
\hline 62500 & $605^{1 \dot{6}}$ & 58564 & $53^{824}$ & $\begin{array}{c}144 \\
48400\end{array}$ & $4243^{6}$ & $\begin{array}{c}178 \\
36865\end{array}$ & $\begin{array}{c}185 \\
34225\end{array}$ \\
\hline 8100 & $980 \mathrm{I}$ & $1 \mp 664$ & I 5876 & 20736 & 26244 & 31684 & 34225 \\
\hline 70600 & $703 x_{7}$ & 70228 & 69700 & 69136 & 68680 & 68549 & $6845^{\circ}$ \\
\hline 0,0595 & 0,0637 & $0,065 \mathrm{I}$ & 0,0732 & 0,0819 & 0,0893 & 0,0912 & 0,0928 \\
\hline
\end{tabular}

ist. In den Punkten findet eine sprungweise Änderung des Neigungswinkels statt, und wir können für die den Punkten entsprechenden Stellungen voraussagen, daß hier die Kurve für den Streukoeffizienten Doppeltangenten hat. Wir haben ferner nur nötig, den Verlauf eines der Koeffizienten, beispielsweise $\mathrm{M}_{1}$, von $\alpha=0$ bis $\alpha=\pi / 2$ zu ermitteln. Der gebrochene Linienzug spiegelt sich in den Ebenen senkrecht zur Ebene durch $\mathrm{M}_{1}$ in den Ordinaten für $\alpha=0$ und $\alpha=\pi / 2$. Die Kurve für den Koeffizienten $M_{2}$ ist aus Symmetriegründen dieselbe wie für $\mathrm{M}_{1}$ (man überzeuge sich durch Vertauschung der Phasen), nur ist sie gegen die erste um $90^{0}$ versetzt, vergleiche die graphische Darstellung der Kolonne $\sigma$ obiger Tabelle, wobei $\sigma$ nach der auf S. 365 angegebenen Formel, unter Benutzung der graphischen Darstellung der Werte für M (Fig. I) berechnet ist.

Da in den Formeln für die Streukoeffizienten die Koeffizienten der Induktion nur als Verhältnisse auftreten, so genügt es für die Bestimmung von $\tau_{1}, \tau_{2}$ und $\sigma$ als reine Zahl, sie nur als allgemeine, d. h. in einem beliebigen Maßstabe geleseneFaktoren anzugeben; die Spulenfaktoren $\mathrm{f}$ sind dann in ihnen implicite enthalten.

Anstatt rechnerisch kann man natürlich bei bereits durch eine Anzahl von Punkten festgelegter Kurve für $\mathrm{M}_{1}$ und $\mathrm{M}_{2}$ die Ermittlung der Streukoeffizienten für beliebige Rotorstellungen auch graphisch vornehmen. (Näheres hierüber siehe Dissertation.)

3. Einige Ergebnisse und deren Diskussion. Für eine Reihe von Nuten-

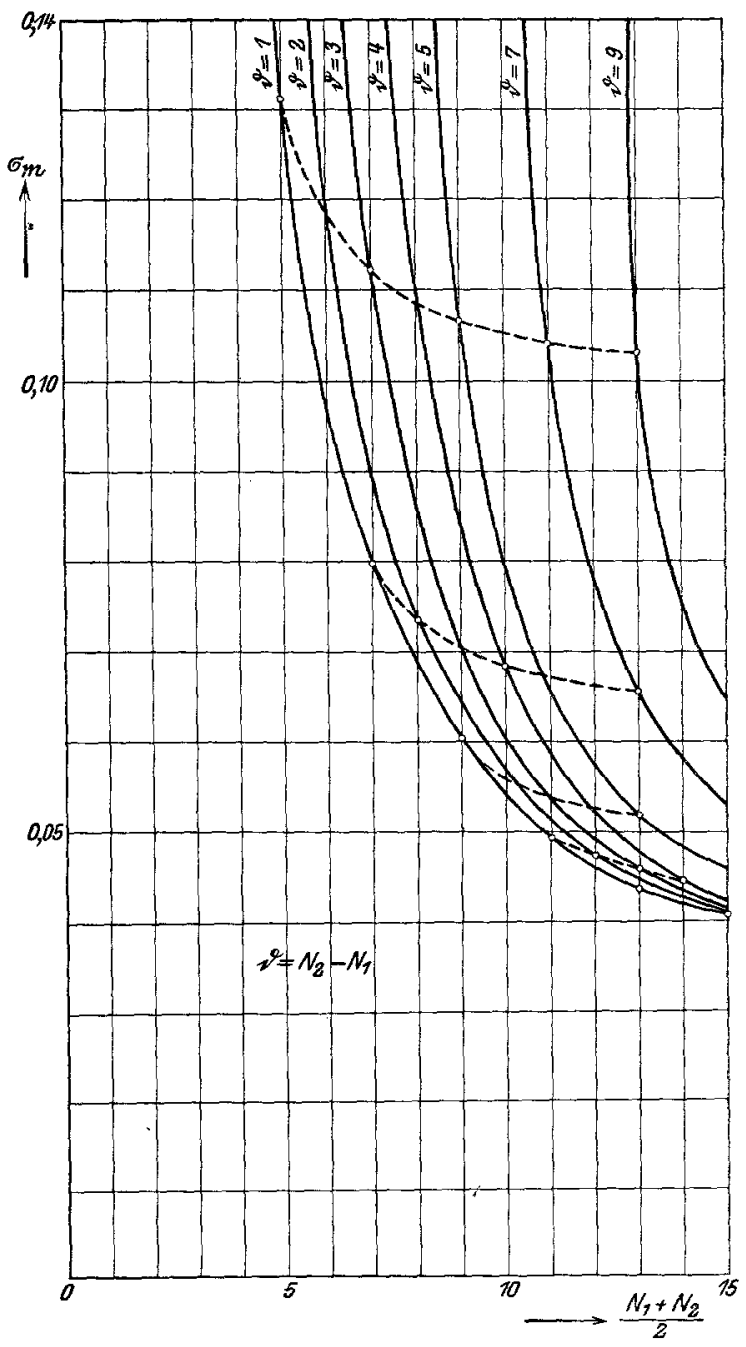

Fig. 2. zahlen ist nach dem Vorhergehenden der Verlauf des Streukoeffizienten $\sigma$ als Funktion des Stator bzw. Rotorumfanges berechnet, doch sind, unter Berücksichtigung der Anlaufsmöglichkeit, nur Maschinen behandelt, deren primäre und sekundäre Nutenzahlen keinen gemeinschaftlichen Teiler besitzen ${ }^{1}$.

1) Im folgenden sind einfach dieMotoren durch das Verhältnis der Nutenzahlen bezeichnet, und zwar bedeutet z. B., $7 / 8$ einen Motor mit $7 \mathrm{Nuten} \mathrm{im}$ Stator und 8 Nuten im Rotor pro Pol und Phase. 


\begin{tabular}{|c|c|c|c|c|c|c|c|}
\hline \multirow{2}{*}{$\mathrm{N}_{1} / \mathrm{N}_{2}$} & \multirow{2}{*}{$=$} & \multicolumn{6}{|c|}{$\mathrm{d}=\mathrm{N}_{2}-\mathrm{N}_{1}=\mathrm{I}$} \\
\hline & & $2 / 3$ & $3 / 4$ & $4 / 5$ & $5 / 6$ & $6 / 7$ & $.7 / 8$ \\
\hline Einfacher Mittelwert & $\approx$ & 0,1389 & 0,0818 & 0,0595 & 0,0490 & $0,043 \mathrm{I}$ & 0,0396 \\
\hline Planimetrischer Mittelwert & $=$ & $0,13 X I$ & $0,08 \pm 0$ & 0,0604 & 0,0493 & 0,0436 & 0,0408 \\
\hline Fehler in $\%$ & $=$ & $-5,4$ & $-0,98$ & $+1,5$ & $0,6 \mathrm{r}$ & $I, I$ & 2,9 \\
\hline
\end{tabular}

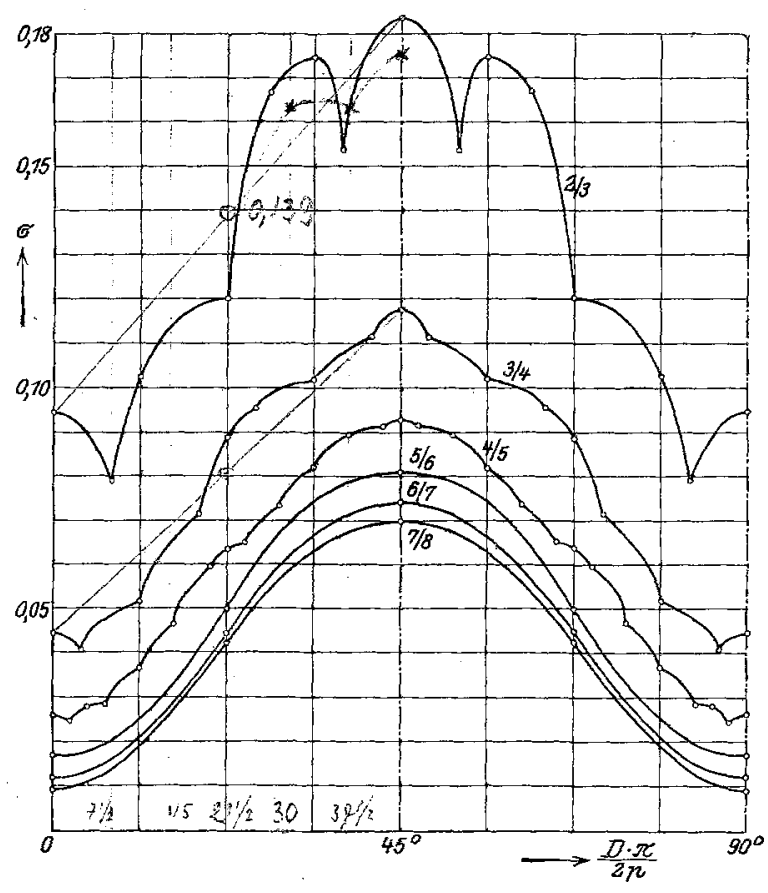

Fig. 3.

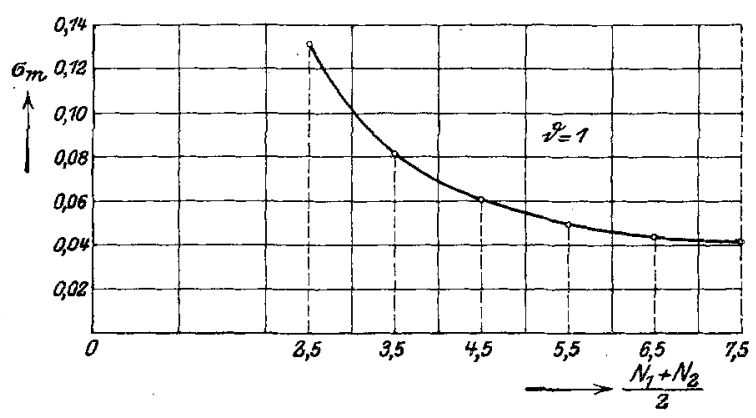

Fig. 4 .

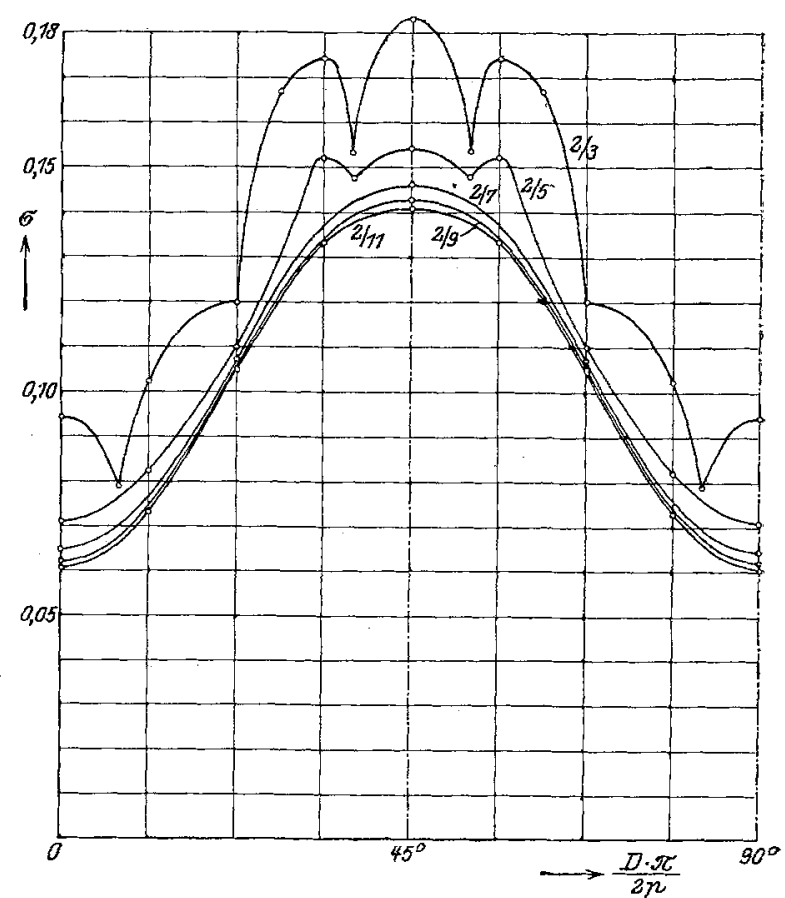

Fig. 5 .

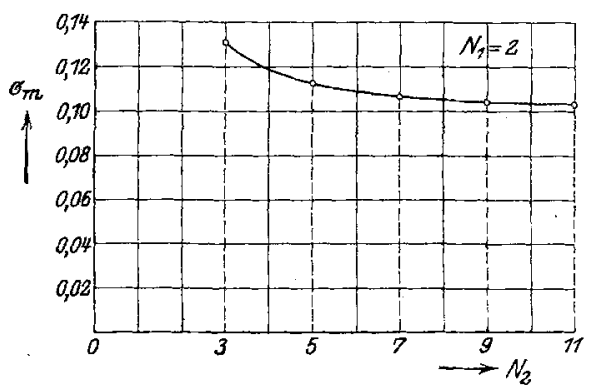

Fig. 6.

Da ferner mit zunehmenden Nutenzahlen die Anzahl der Doppelpunkte der Kurve stark wächst, und gleichzeitig eine große Näherung an eine stetige Kurve stattfindet, so sind nur die Motoren $2 / 3,3 / 4$ and $4 / 5$ exakt behandelt 1 ). Bei den übrigen sind zwischen $\alpha=0$ und $\alpha=90^{\circ}$ fünf bis neun Punkte bestimmt und durch eine stetige Kurve verbunden. Deren Mittelwert ist planimetrisch erhalten und in Beziehung gebracht zum einfachen Mittelwert von Rogowski und Simons. Die angegebenen Fehler beziehen sich stets auf den planimetrischen Mittelwert.

1) Bereits bei $4 / 5$ ist der Fehler zwischen exakter Kurve und Kurve durch 9 Punkte unmerklich größer als die Fehlergrenze beim Planimetrieren. 


\begin{tabular}{|c|c|c|c|c|c|c|c|c|c|c|}
\hline \multicolumn{2}{|c|}{$\mathrm{d}=2$} & \multicolumn{2}{|c|}{$\mathrm{d}=3$} & \multicolumn{2}{|c|}{$d=4$} & \multicolumn{2}{|c|}{$\mathrm{d}=5$} & \multicolumn{2}{|c|}{$d=7$} & $\mathrm{~d}=9$ \\
\hline $3 / 5$ & $5 / 7$ & $2 / 5$ & $5 / 8$ & $3 / 7$ & $5 / 9$ & $2 / 7$ & $4 / 9$ & $2 / 9$ & $3 / 10$ & $2 / 11$ \\
\hline 0,0720 & $0,0_{4} 6 I$ & o, I I 28 & 0,0453 & 0,0665 & $0,043^{8}$ & 0,1055 & 0,0509 & 0,1025 & 0,0639 & 0,1009 \\
\hline 0,0736 & $0,047^{6}$ & 0,1123 & $0,045^{8}$ & 0,0684 & 0,0445 & 0,1066 & 0,0519 & $0, \mathrm{IO}_{4} \mathrm{I}$ & 0,0655 & 0,1030 \\
\hline 2,2 & 3 & $-0,45$ & $\mathrm{I}, \mathrm{I}$ & 3 & I, 6 & 1,03 & 2 & $\mathrm{I}, 5$ & 2,4 & 2 \\
\hline
\end{tabular}

In der Figur 2 sind alle in der beigegebenen Tabelle eingetragenen Rechnungsergebnisse graphisch veranschaulicht. Dieausgezogenen Kurven stellen den planimetrischen Mittelwert desStreukoeffizientenals Funktion der mittleren Nutenzahl dar, die gestrichelten Kurven verbinden Punkte gleicher primärer Nutenzahl.

In den beiden folgenden Figuren sind die Kurven der Streukoeffizienten für einige Motoren mit dem Unterschiede d = I zwischen primärer und sekundärer Nutenzahl und, um die Abnahme der doppelt verketteten Streuung mit der Zunahme der Nutenzahl deutlich zu zeigen, die planimetrischen Mittelwerte als Funktion der mittleren Nutenzahl gezeichnet.

Läßt man bei ein und derselben primären Nutenzahl lediglich die sekundäre Nutenzahl wachsen, so findet man, daß sich die Kurven für den Streukoeffizienten rasch nähern, der Mittelwert also einer Konstanten zustrebt; dies geht deutlich aus den beiden Figuren 5 und 6 hervor, welche die Kurven für. $\sigma$ für die Motoren $2 / 3,2 / 5,2 / 7,2 / 9,2 / 11$,

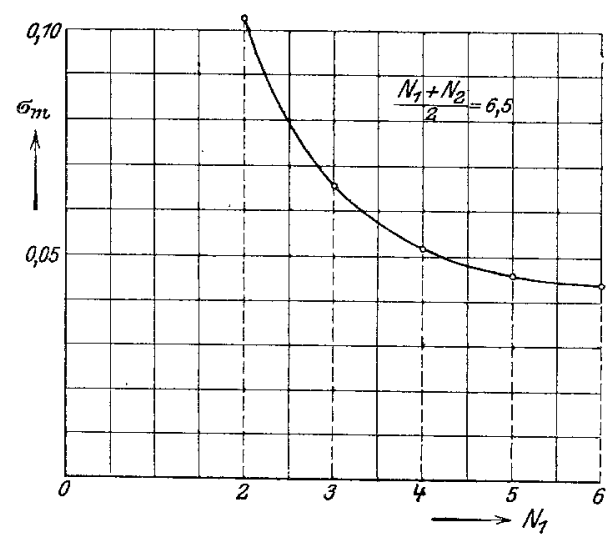

Fig. 7 .

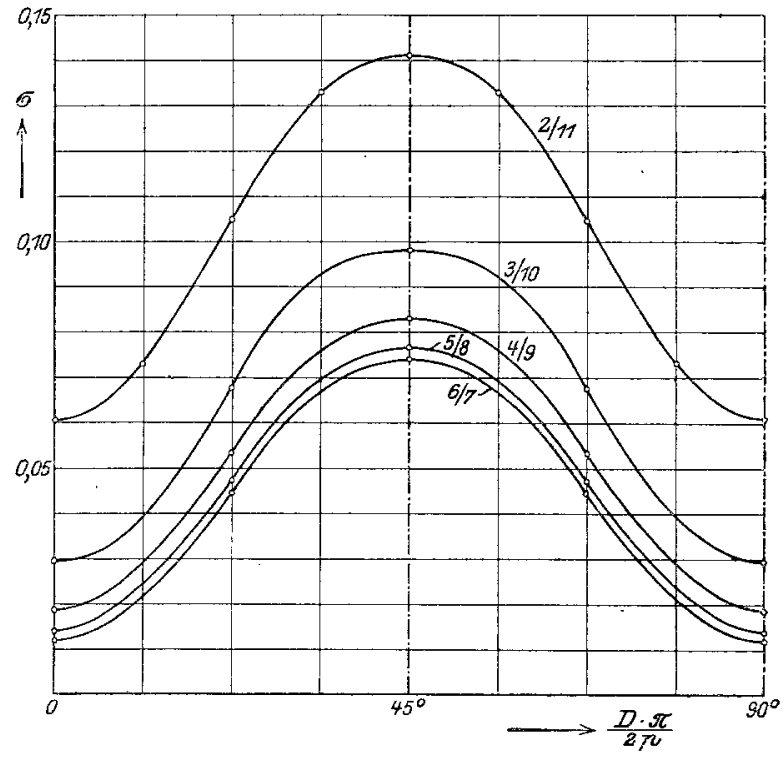

Fig. 8.

bzw. den planimetrischen Mittelwert als Funktion der selkundären Nutenzahl darstellen.

Verfehlt ist es jedoch, wollte man einem Vorschlage von Hellmund folgen und von vornherein die Streukoeffizienten für eine mittlere Nutenzahl berechnen. Greifen wir z. B. die mittlere Nutenzahl 6,5 heraus, die durch die Verhältnisse

$$
2 / 11,3 / 10,4 / 9,5 / 8 \text { und } 6 / 7
$$

erreicht wird. Wäre Hellmunds Vorgehen richtig, so müßte eine Rechnung nach dem Vorhergehenden für alle diese Motoren wenigstens in genügender Annäherung die gleichen Werte liefern. Die Betrachtung der Tabelle und der Figuren 7 und 8 zeigt jedoch, daß dies nicht der Fall ist.

Das Ergebnis unserer Untersuchungen können wir dahin zusammenfassen, daß die Verhältnisse bezüglich der doppelt verketteten Streuung umso günstiger werden, je größer 
die Anzahl der Nuten primär und sekundär, je kleiner die Differenz d beider Nutenzahlen, bezogen auf die größere, ist. Bei gleicher primärer Nutenzahl nimmt der Koeffizient der doppelt verketteten Streuung etwas mit der Zunahme der sekundären Nutenzahl ab; für eine mittlere Nutenzahl ist die Differenz der primären und sekundären Nutenzahl bestimmend für die Größe der doppelt verketteten Streuung.

Beim Übergang des Rotors von einer Hauptstellung in die andere ändert sich der Koeffizient der doppelt verketteten Streuung nicht stetig, sondern die zugehörige Kurve weist - der Annahme treppenförmiger Felder gemäß - eine Anzahl von Doppelpunkten auf. Diese Anzahl wächst sehr schnell mit zunehmender Nutenzahl, und gleichzeitig nähert sich die Kurve mehr und mehr einer stetigen. Während man bei kleineren Nutenzahlen den Verlauf der Kurve kennen muß, wenn man den planimetrischen Mittelwert genau errechnen will, so genügt bei größeren Nutenzahlen die Ermittlung einer verhältnismäßig geringen Anzahl von Punkten, die, durch eine stetige Kurve verbunden, zu einem für alle Bedürfnisse der Praxis ausreichend genauen Näherungswert führen.

Der Vorschlag von Rogowski und Simons, sich auf die Betrachtung der Hauptstellungen zu beschränken, hat mit Rücksicht auf die Bedürfnisse der Praxis Gültigkeit, zum mindesten für ungleiche Nutenzahlen. Ganz unhaltbar hingegen ist der Vorschlag von $\mathrm{Hellmund} \mathrm{d}^{1}$, die Streukoeffizienten von vornherein für eine mittlere Nutenzahl zu errechnen.

\section{Die doppelt verkettete Streuung beim Zwei- und Dreiphasenmotor.}

4. Beziehungen zwischen den Strömen im Stator und im Rotor. Wir behandeln im folgenden die Verhältnisse der doppelt verketteten Streuung eines Motors, welcher dadurch entsteht, daß man in einen Stator mit symmetrischer Drehstromwicklung einen Rotor mit symmetrischer Zweiphasenwicklung einbaut.

Das so entstandene Gebilde ist jetzt nicht mehr vollkommen symmetrisch; wir unterscheiden auch bei diesem Motor wieder zwei ausgezeichnete Stellungen:

In der ,ersten Hauptstellung“" decken sich die Mittellinien je einer Stator- und einer Rotorphase, während gleichzeitig die Mitte der anderen Rotorphase in die Mitte zwischen den übrigen Statorphasen fällt.

In der ,zweiten Hauptstellung“ liegt die Mittellinie zwischen den Rotorphasen in der Mitte zwischen zwei Statorphasen.

Beziehen wir uns - wie üblich - auf einen zweipoligen Motor, rechnen also die Polteilung $\tau \mathrm{zu} \pi=\mathrm{I} 80^{\circ}$, dann ist, um den Rotor aus einer Hauptstellung in die nächstfolgende zu bringen, eine Drehung von $\pi / \mathrm{I} 2=I 5^{0}$ auszuführen.

Wir denken uns jetzt einmal den zur experimentellen Ermittlung des Streukoeffizienten erforderlichen Leerlaufs- und Kurzschlußversuch ausgeführt (siehe I, 2), dem Stator drücken wir eine reine Sinusspannung auf. Für den Fall der Sternschaltung gilt dann, wenn E die Amplitude der Spannungswelle ist:

$$
\begin{aligned}
\mathrm{e}_{\mathrm{a}}-\mathrm{e}_{\mathrm{b}} & =\mathrm{E} \cdot \sin \omega \mathrm{t} \\
\mathrm{e}_{\mathrm{b}}-\mathrm{e}_{\mathrm{c}} & =\mathrm{E} \cdot \sin (\omega \mathrm{t}+\mathrm{.2} 20) \\
\mathrm{e}_{\mathrm{c}}-\mathrm{e}_{\mathrm{a}} & =\mathrm{E} \cdot \sin (\omega \mathrm{t}+240) \\
\Sigma \mathrm{E} & =0 .
\end{aligned}
$$

Bei stillstehendem, geöffnetem Rotor liegen dann die gleichen Verhältnisse wie bei einem Drehstrommotor vor, da wegen Stromlosigkeit der Sekundärteil nichł auf den Primärteil rückwirken kann: die Sinoidalwechselfelder erzeugen ein reines Drehfeld.

Anders beim Kurzschlußversuch. Jetzt können wir deutlich den Einfluß der vorhandenen Unsymmetrie wahrnehmen, wenn wir gleichzeitig die Ströme in den Statorphasen

1) Dies hat Hell mund in einer seiner letzten Arbeiten bereits gleichfalls erkannt. 
messen. Für eine beliebige Stellung des Rotors sind dann die Effektivwerte voneinander verschieden. Da nun für jeden Moment die Summe der Ströme verschwindet, so müssen die Maxima der Phasenströme um einen Winkel gegeneinander verschoben sein, der im allgemeinen von $\frac{2 \pi}{3}=120^{\circ}$ verschieden ist.

Wir wollen zunächst einmal die Beziehungen zwischen den Stromamplituden und Phasenverschiebungen mathematisch festlegen.

$\mathrm{Zu}$ diesem $Z$ wecke beziehen wir alles auf eine Phase, z. B. die Phase a, die dadurch gekennzeichnet sei, daß sie den schwächsten Strom führt. Für den Momentanwert dieses Stromes gilt dann:

$$
i_{a t}=J_{a} \sin \omega t
$$

Bedeuten dann $\Delta \mathrm{J}_{\mathrm{b}}$ und $\Delta \mathrm{J}_{\mathrm{o}}$ diejenigen Größen, um welche die Stromamplituden $\mathrm{J}_{\mathrm{b}}$ und $\mathrm{J}_{\mathrm{c}}$ von $\mathrm{J}_{\mathrm{a}}$ abweichen, sind ferner $\mathrm{b}$ und $\mathrm{c}$ kleine, zusätzliche Winkel, welche zusammen mit $\frac{2 \pi}{3}$ bzw. $\frac{4 \pi}{3}$ die zeitliche Verschiebung gegen $\mathrm{J}_{\mathrm{a}}$ bestimmen, dann haben wir zu schreiben:

$$
\begin{aligned}
& \dot{i}_{b t}=\left\{J_{a}+\Delta J_{b}\right\} \cdot \sin (\omega t+\mathrm{I} 20+b) \\
& i_{c t}=\left\{J_{a}+\Delta J_{c}\right\} \cdot \sin (\omega t+240+c) .
\end{aligned}
$$

Die Aufstellung dieser Gleichungen ist nichts weiter als die Herstellung der bekannten Beziehung

$$
\Sigma i_{t}=0 \text {. }
$$

Durch Ausrechnung und Einführen der Grenzwerte der sin und cos der kieinen Winkel $\mathrm{b}$ und $\mathrm{c}$ sowie durch Vernachlässigung der Produkte $\Delta \mathrm{J}_{\mathrm{b}} \cdot \sin \mathrm{b}$ und $\Delta \mathrm{J}_{\mathrm{c}} \cdot \sin \mathrm{c}$ wird man auf folgende Gleichungen

$$
\begin{aligned}
& -\mathrm{J}_{\mathrm{a}} \cdot \mathrm{b}+\sqrt{3} \Delta \mathrm{J}_{\mathrm{b}}-\mathrm{J}_{\mathrm{a}} \cdot \mathrm{c}-\sqrt{3} \Delta \mathrm{J}_{\mathrm{c}}=0 \\
& -\sqrt{3} \mathrm{~J}_{\mathrm{a}} \cdot \mathrm{b}-\Delta \mathrm{J}_{\mathrm{b}}+\sqrt{3} \mathrm{~J}_{\mathrm{a}} \cdot \mathrm{c}-\Delta \mathrm{J}_{\mathrm{c}}=0 .
\end{aligned}
$$

geführt, aus denen sich, da $\Delta \mathrm{J}_{\mathrm{b}}$ und $\Delta \mathrm{J}_{\mathrm{c}}$ aus Amperemeterablesungen bestimmt werden die zusätzlichen Winkel $\mathrm{b}$ und $\mathrm{c}$ folgendermaßen errechnen:

$$
\mathrm{b}=\frac{\Delta \mathrm{J}_{\mathrm{b}}-2 \Delta \mathrm{J}_{\mathrm{c}}}{\sqrt{3} \mathrm{~J}_{\mathrm{a}}} ; \quad \mathrm{c}=\frac{2 \Delta \mathrm{J}_{\mathrm{b}}-\Delta \mathrm{J}_{\mathrm{c}}}{\sqrt{3} \mathrm{~J}_{\mathrm{a}}} \text {. }
$$

Aus der Betrachtung der gewonnenen Formeln erhellen folgende Beziehungen, die allgemein gültig sind:

$$
\begin{aligned}
& \text { Für } \mathrm{J}_{a}+\mathrm{J}_{\mathrm{b}}-2 \mathrm{~J}_{0} \sum_{<} \quad \text { wird } \mathrm{b} \gtreqless 0 \\
& \text {, } \mathrm{J}_{\mathrm{a}}+\mathrm{J}_{\mathrm{c}}-2 \mathrm{~J}_{\mathrm{b}} \gtreqless 0 \quad, \quad \mathrm{c} \varliminf_{0} .
\end{aligned}
$$

Wir erkennen, daß es jetzt aus Mangel an vollkommener Symmetrie keinen einheitlich definierten Kurzschlußstrom mehr gibt. Man würde sich etwa dazu entschließen können, den bei ganz langsamer Drehung des Rotors zu messenden mittleren Effektivwert, der für alle Phasèn natürlich der gleiche ist, für die Auftragung des Heylandschen Kreisdiagrammes als Grundlage zu nehmen. Für die rechnerische Bestimmung des Streukoeffizienten besteht dann die Schwierigkeit, diesen Mittelwert des Kurzschlußstromes aus den Gleichungen zu berechnen.

Bevor wir jedoch auf diesen Umstand näher eingehen, wollen wir die vorstehenden Betrachtungen über die primären Ströme auf die sekundären Ströme ausdehnen.

Bedeutet w den Widerstand einer Rotorphase, und werden die Gegeninduktivitäten durch $\mathfrak{m}$ gekennzeichnet, dann haben wir für die sekundären Spannungen bei Stillstand und Kurzschluß die Gleichungen: 


$$
\begin{aligned}
& e_{x t}=o=w \cdot i_{x t}+\mathfrak{M}_{a x} \frac{d i_{a t}}{d t}+\mathfrak{M}_{b x} \frac{d i_{b t}}{d t}+\mathfrak{M}_{c x} \frac{d i_{c t}}{d t} \\
& e_{y t}=0=w \cdot i_{y t}+M_{a y} \frac{d i_{a t}}{d t}+M_{b y} \frac{d i_{b t}}{d t}+M_{c y} \frac{d i_{c t}}{d t}
\end{aligned}
$$

Ist die zeitliche Verschiebung des Stromes $i_{x t}$ gegenüber dem Strom $i_{a t}$ in der Bezugsphase a durch den Winkel $\psi$ gegeben, und sind ferner $\Delta \mathrm{J}_{\mathrm{x}}$ und $\Delta \mathrm{J}_{\mathrm{y}}$ die Größen, um welchem die sekundären Stromamplituden von $J_{a}$ verschieden sind, bedeutet endlich y einen kleinen zusätzlichen Winkel, welcher der Abweichung gegenüber reinem Zweiphasenstrom Rechnung trägt, so müssen wir für die sekundären Ströme den Ansatz machen:

$$
\begin{aligned}
& i_{x t}=\left\{J_{a}+\Delta J_{x}\right\} \cdot \sin (\omega t+\psi) \\
& i_{y t}=\left\{J_{a}+\Delta J_{y}\right\} \cdot \cos (\omega t+\psi+y) .
\end{aligned}
$$

Unter Berücksichtigung der Beziehungen für die Momentanwerte der primären Ströme kann man jetzt zwei Gleichungen hinschreiben, welche durch Weiterentwicklung in zwei Ausdrücke übergehen, aus denen man die zur Bestimmung der vier Unbekannten $\psi, \Delta J_{x}$, $\Delta \mathrm{J}_{\mathrm{y}}$ und y erforderlichen vier Gleichungen leicht ableiten kann, wenn man, wie vorhin, die Produkte kleiner Größen vernachlässigt, für den sin und cos der kleinen Winkel ihre Grenzwerte für o einsetzt und die Sinus- und Cosinusglieder zusammenfaßt ${ }^{\mathbf{1}}$ ).

Die auf diese Weise zu errechnenden Resultate lauten folgendermaßen:

$$
\begin{aligned}
& \operatorname{tg} \psi=\frac{2 \mathfrak{M}_{\mathrm{ax}} \cdot \mathrm{J}_{\mathrm{a}}-\mathfrak{M}_{\mathrm{bx}}\left\{\mathrm{J}_{\mathrm{a}}(\mathrm{I}+\sqrt{3} \mathrm{~b})+\Delta \mathrm{J}_{\mathrm{b}}\right\}-\mathfrak{M}_{\mathrm{cx}}\left\{\mathrm{J}_{\mathrm{a}}(\mathrm{I}-\sqrt{3} \cdot \mathrm{c})+\Delta \mathrm{J}_{\mathrm{c}}\right\}}{\mathfrak{M}_{\mathrm{bx}}\left\{\mathrm{J}_{\mathrm{a}}(\sqrt{3}+\mathrm{b})+\sqrt{3} \Delta \mathrm{J}_{\mathrm{b}}\right\}+\mathfrak{M}_{\mathrm{cx}}\left\{\mathrm{J}_{\mathrm{a}}(\sqrt{3}+\mathrm{c})+\sqrt{3} \Delta \mathrm{J}_{\mathrm{c}}\right\}} \\
& \Delta J_{x}=\frac{\frac{\omega}{2} \mathscr{M}_{b x}\left\{\sqrt{3} \cdot\left(J_{a}+\Delta J_{b}\right)-J_{a} \cdot b\right\}-\frac{\omega}{2} \mathbb{M}_{\mathrm{cx}}\left\{J_{a} \cdot c+\sqrt{3} \cdot\left(J_{a}+\Delta J_{c}\right)\right\}-w J_{a} \cdot \cos \psi}{w \cdot \cos \psi} \\
& \Delta \mathrm{J}_{\mathrm{y}}=-\frac{\mathrm{I}}{\mathrm{w}}\left\{\mathrm{w} \cdot \mathrm{J}_{\mathrm{a}}+\frac{\omega}{2}\left[\operatorname { s i n } \psi \left\{\mathfrak{M}_{\mathrm{by}}\left[\mathrm{J}_{\mathrm{a}} \cdot \mathrm{b}-\sqrt{3}\left(\mathrm{~J}_{\mathrm{a}}+\Delta \mathrm{J}_{\mathrm{b}}\right)\right]\right.\right.\right. \\
& \left.+\mathfrak{M}_{\mathrm{cy}}\left[\mathrm{J}_{\mathrm{a}} \cdot \mathrm{c}+\sqrt{3}\left(\mathrm{~J}_{\mathrm{a}}+\Delta \mathrm{J}_{\mathrm{c}}\right)\right]\right\}+\cos \psi\left\{2 \mathfrak{M}_{\mathrm{ay}} \mathrm{J}_{\mathrm{a}}\right. \\
& \left.\left.\left.-\mathfrak{M}_{b y}\left[\left(J_{a}+\Delta J_{b}\right)+\sqrt{3} J_{a} \cdot b\right]+\mathfrak{M}_{c y}\left[\sqrt{3} J_{a} \cdot c-\left(J_{a}+\Delta J_{c}\right)\right]\right\}\right]\right\} \\
& \mathrm{y}=\frac{\mathrm{w} \cdot\left\{\mathrm{J}_{\mathrm{a}}+\Delta \mathrm{J}_{\mathrm{y}}\right\} \cos \psi+\omega \mathfrak{M}_{\mathrm{a} y} \cdot \mathrm{J}_{\mathrm{a}}-\frac{\mathrm{I}}{2} \omega \mathfrak{M}_{\mathrm{b}_{\mathrm{y}}}\left\{\mathrm{J}_{\mathrm{a}}+\Delta \mathrm{J}_{\mathrm{b}}\right\}}{\mathrm{w} \cdot \mathrm{J}_{\mathrm{a}} \cdot \sin \psi} \\
& +\frac{-\frac{\sqrt{3}}{2} \omega M_{\mathrm{by}} \mathrm{J}_{\mathrm{a}} \cdot \mathrm{b}-\frac{\mathrm{I}}{2} \omega \mathfrak{M}_{\mathrm{c} y}\left\{\mathrm{~J}_{\mathrm{a}}+\Delta \mathrm{J}_{\mathrm{c}}\right\}+\frac{\sqrt{3}}{2} \omega \mathfrak{M}_{\mathrm{cy}} \mathrm{J}_{\mathrm{a}} \cdot \mathrm{c}}{\mathrm{w} \cdot \mathrm{J}_{\mathrm{a}} \sin \psi} .
\end{aligned}
$$

Unter Benutzung dieser Gleichungen im Verein mit den vorstehend angeführten ist man nun imstande, ein etwaiges vollständiges Spannungs- und Stromdiagramm für den vorliegenden Motor zu zeichnen.

5. Berechnung der Streukoeffizienten aus den Leerlaufsgleichungen. Entsprechend den Blondelschen Definitionen ${ }^{2}$ ) der einfachen Streukoeffizienten lösen wir zunächst die Gleichungen für gespeisten Stator und stillstehenden, geöffneten Rotor, sodann für den Fall gespeisten Sekundärteiles und offenen Primärteiles.

Unserer Voraussetzung gemäß zwingen wir dem Stator eine reine Sinuswelle auf, so daß wir, wenn wieder W den Widerstand einer Primärphase, L deren Eigeninduktivität

1) Näheres siehe Dissertation.

9) Vgl. unter 1 . 
und $\mathfrak{M}$ die primäre Gegeninduktivität bezeichnet, unter Beibehaltung der sonst üblichen Schreibweise zu setzen haben:

$$
\begin{aligned}
& e_{a}=W \cdot i_{a}+(L-M) \frac{d i_{a}}{d t} \\
& e_{b}=W \cdot i_{b}+(L-M) \frac{d i_{b}}{d t} \\
& e_{c}=W \cdot i_{c}+(L-M) \frac{d i_{c}}{d t}
\end{aligned}
$$

Die Bedingung der Gültigkeit der Anwendung der Kirchhoffschen Regel zur Vorzeichenbestimmung der elektromotorischen Kräfte verlangt, daß man über das Vorzeichen von $\mathfrak{M}$ bei Dreiphasenstrom eine Voraussetzung macht ${ }^{1}$ ), je nachdem man nämlich $\mathfrak{M}$ als eine von den räumlichen Beziehungen des Gebildes unabhängige, positive Zahl aufzufassen wünscht, oder ob man $\mathfrak{M}$ selbst von positiven Werten durch Null hindurch zu negativen, und umgekehrt, wandern lassen will. Wir treffen die letztere Festsetzung und schreiben weiter für Sternschaltung:

$$
\begin{aligned}
& \mathrm{e}_{\mathrm{a}}-\mathrm{e}_{b}=\mathrm{E}_{1} \sin \omega \mathrm{t} \\
& \mathrm{e}_{b}-\mathrm{e}_{\mathrm{c}}=\mathrm{E}_{1} \sin (\omega \mathrm{t}+\mathrm{I} 20) \\
& \mathrm{e}_{\mathrm{c}}-\mathrm{e}_{\mathrm{a}}=\mathrm{E}_{1} \sin (\omega \mathrm{t}+240)
\end{aligned}
$$

Für die primären Ströme, die nach den Gleichungen ebenfalls einfache Sinusfunktionen sind, setzen wir:

$$
\begin{aligned}
& \mathrm{i}_{\mathrm{a}}=\mathrm{J}_{1} \cdot \sin \omega \mathrm{t} \\
& \mathrm{i}_{\mathrm{b}}=\mathrm{J}_{1} \cdot \sin (\omega \mathrm{t}+\mathrm{I} 20) \\
& \mathrm{i}_{\mathrm{c}}=\mathrm{J}_{1} \cdot \sin (\omega \mathrm{t}+240) .
\end{aligned}
$$

Die Lösung dieser Drehstromgleichungen lautet:

wo:

$$
\mathrm{E}_{1} \cdot \sin \omega \mathrm{t}=\mathrm{J}_{1} \cdot \sqrt{\mathrm{W}^{2}+\omega^{2}(\mathrm{~L}-\mathfrak{M})^{2}} \cdot \sin (\omega \mathrm{t}+\varphi)
$$

$$
\operatorname{tg} \varphi=\frac{W-\sqrt{3} \omega(\mathrm{L}-\mathfrak{M})}{\sqrt{3} W+\omega(\mathrm{L}-\mathfrak{M})} .
$$

Die Effektivwerte sind also gegeben durch:

$$
\overline{\mathrm{E}_{1}}=\overline{\mathrm{J}_{1}} \cdot \sqrt{\mathrm{W}^{2}+\omega^{2}(\mathrm{~L}-\mathfrak{M})^{2}} \text {. }
$$

Die Gleichungen für die sekundär induzierten Spannungen heißen:

$$
\begin{aligned}
& \mathrm{e}_{\mathrm{x}}=\mathfrak{M}_{\mathrm{ax}} \frac{\mathrm{di_{ \textrm {a } }}}{\mathrm{dt}}+\mathfrak{M}_{\mathrm{bx}} \cdot \frac{\mathrm{di}}{\mathrm{dt}}+\mathfrak{M}_{\mathrm{cx}} \frac{\mathrm{di}_{\mathrm{c}}}{\mathrm{dt}} \\
& \mathrm{e}_{\mathrm{y}}=\mathfrak{M}_{\mathrm{ay}} \cdot \frac{\mathrm{di}}{\mathrm{dt}}+\mathfrak{M}_{\mathrm{by}} \cdot \frac{\mathrm{di}}{\mathrm{dt}}+\mathfrak{M}_{\mathrm{cy}} \cdot \frac{\mathrm{di}}{\mathrm{dt}}
\end{aligned}
$$

und führen zu der Lösung:

$$
\left.\mathrm{E}_{2}=\omega \cdot \mathrm{J}_{1} \cdot \sqrt{\left\{\mathfrak{M}_{\mathrm{ax}}-\frac{\mathrm{I}}{2} \mathfrak{M}_{\mathrm{bx}}-\frac{\mathrm{I}}{2} \mathfrak{M}_{\mathrm{cx}}\right\}^{2}+\frac{3}{4}\left\{\mathfrak{M}_{\mathrm{cx}}-\mathfrak{M}_{\mathrm{bx}}\right\}^{2}} \cdot \sin (\omega t+\psi)^{2}\right)
$$

1) Vg1. die Arbeit von Rogowski und Simons, ETZ. I9o9, Heft ro und II.

2) Siehe auch Rogowski und Simons a. a. O. 
Die zeitliche Verschiebung \& wird gefunden aus:

$$
\operatorname{tg} \psi=\frac{\mathfrak{M}_{\mathrm{ax}}-\frac{I}{2} \mathfrak{M}_{\mathrm{bx}}-\frac{I}{2} \mathfrak{M}_{\mathrm{cx}}}{\frac{\sqrt{3}}{2}\left\{\mathfrak{M}_{\mathrm{cx}}-\mathfrak{M}_{\mathrm{bx}}\right\}} .
$$

Für die Effektivwerte ist also zu schreiben:

$$
\overline{\mathrm{E}_{2}}=\omega \overline{J_{1}} \cdot \sqrt{\left.\left\{\mathfrak{M}_{\mathrm{ax}}-\frac{\mathrm{I}}{2} \mathfrak{M}_{\mathrm{bx}}-\frac{\mathrm{I}}{2} \mathfrak{M}_{\mathrm{cx}}\right\}^{2}+\frac{3}{4}\left\{\mathfrak{M}_{\mathrm{cx}}-\mathfrak{M}_{\mathrm{bx}}\right\}^{2}{ }^{1}\right)} .
$$

Jetzt vertauschen wir die Rollen primär und sekundär, führen also dem Rotor bei geöfnetem Stator reinen Zweiphasenstrom zu. Bedeuten wieder w und 1 den Widerstand bzw. die Eigeninduktivität einer sekundären Phase, dann haben wir die Beziehungen:

$$
\begin{aligned}
& \mathrm{e}_{\mathrm{xt}}^{\prime}=\mathrm{J}_{2} \cdot \mathrm{w} \cdot \sin \omega t+1 \frac{\mathrm{d}\left[\mathrm{J}_{2} \cdot \sin \omega \mathrm{t}\right]}{\mathrm{dt}} \\
& \mathrm{e}_{\mathrm{yt}}^{\prime}=\mathrm{J}_{2} \cdot \mathrm{w} \cos \omega \mathrm{t}+1 \cdot \frac{\mathrm{d}\left[\mathrm{J}_{2} \cdot \cos \omega \mathrm{t}\right]}{\mathrm{dt}} \\
& \mathrm{e}_{\mathrm{at}}^{\prime}=\mathfrak{M}_{\mathrm{ax}} \frac{\mathrm{d}\left[\mathrm{J}_{2} \cdot \sin \omega \mathrm{t}\right]}{\mathrm{dt}}+\mathfrak{M}_{\mathrm{ay}} \cdot \frac{\mathrm{d}\left[\mathrm{J}_{2} \cos \omega \mathrm{t}\right]}{\mathrm{dt}} \\
& \mathrm{e}_{\mathrm{bt}}^{\prime}=\mathfrak{M}_{\mathrm{bx}} \frac{\mathrm{d}\left[\mathrm{J}_{2} \cdot \sin \omega \mathrm{t}\right]}{\mathrm{dt}}+\mathfrak{M}_{\mathrm{by}} \cdot \frac{\mathrm{d}\left[\mathrm{J}_{2} \cdot \cos \omega \mathrm{t}\right]}{\mathrm{dt}} \\
& \mathrm{e}_{\mathrm{ct}}^{\prime}=\mathfrak{M}_{\mathrm{cx}} \cdot \frac{\mathrm{d}\left[\mathrm{J}_{2} \cdot \sin \omega \mathrm{t}\right]}{\mathrm{dt}}+\mathfrak{M}_{\mathrm{cy}} \cdot \frac{\mathrm{d}\left[\mathrm{J}_{2} \cdot \cos \omega \mathrm{t}\right]}{\mathrm{dt}} .
\end{aligned}
$$

Aus diesen Gleichungen ergeben sich folgende Resultate:

$$
\begin{aligned}
& \mathrm{E}_{2 t}^{\prime}=\mathrm{J}_{2} \cdot \sqrt{\mathrm{w}^{2}+\omega^{2} \mathrm{l}^{2}} \cdot \sin (\omega \mathrm{t}+\chi) \\
& \operatorname{tg} \chi=\frac{\omega 1}{w} \\
& \overline{\mathrm{E}_{2}^{\prime}}=\overline{\mathrm{J}}_{2} \cdot \sqrt{\mathrm{w}^{2}+\omega^{2} 1^{2}} \\
& E_{i t}^{\prime}=\omega \cdot J_{2} \sqrt{M_{a x}^{2}+\mathfrak{M}_{a y}^{2}} \cdot \cos (\omega t+\xi) \\
& \operatorname{tg} \xi=-\frac{M_{\mathrm{ax}}}{\mathfrak{M}_{\mathrm{ax}}} \\
& \overline{\mathrm{E}_{1}^{\prime}}=\omega \overline{\mathrm{J}_{2}} \sqrt{\mathfrak{M}_{\mathrm{ax}}{ }^{2}+\bar{M}_{\mathrm{ay}^{2}}} .
\end{aligned}
$$

Für die Errechnung der Streukoeffizienten sind nur die ideellen Ströme zu berücksichtigen, welche sich durch Vernachlässigung der Ohmschen Widerstände aus obigen Gleichungen ergeben. Wir bekommen:

$$
\begin{aligned}
\tau_{1}=\frac{\overline{E_{1}}-\overline{E_{2}}}{\overline{E_{2}}}=\frac{\left\{L-M_{M}-\sqrt{\left(\mathfrak{M}_{\mathrm{ax}}-\frac{\mathrm{I}}{2} \mathfrak{M}_{\mathrm{bx}}-\frac{\mathrm{I}}{2} \mathfrak{M}_{\mathrm{cx}}\right)^{2}+\frac{3}{4}\left(\mathfrak{M}_{\mathrm{cx}}-\mathfrak{M}_{\mathrm{bx}}\right)^{2}}\right.}{\sqrt{\left(\mathfrak{M}_{\mathrm{ax}}-\frac{\mathrm{I}}{2} \mathfrak{M}_{\mathrm{bx}}-\frac{\mathrm{I}}{2} \mathfrak{M}_{\mathrm{cx}}\right)^{2}+\frac{3}{4}\left(\mathfrak{M}_{\mathrm{cx}}-\mathfrak{M}_{\mathrm{bx}}\right)^{2}}} \\
\tau_{2}=\frac{\overline{\mathrm{E}_{2}^{\prime}}-\overline{\mathrm{E}_{\mathrm{I}}^{\prime}}}{\overline{\mathrm{E}_{1}^{\prime}}}=\frac{I-\sqrt{\mathfrak{M}_{\mathrm{ax}}{ }^{2}+\mathfrak{M}_{\mathrm{ay}}^{2}}}{\sqrt{\mathfrak{M}_{\mathrm{ax}}^{2}+\mathfrak{M}_{\mathrm{ay}}{ }^{2}}}
\end{aligned}
$$

1) Siehe auch Rogowski und Simons a. a. O. 
Hiernach lautet der Ausdruck für den Heylandschen Streukoeffizienten:

$$
\begin{aligned}
& \sigma= \tau_{1}+\tau_{2}+\tau_{1} \cdot \tau_{2}= \\
& \frac{\{\mathrm{L}-\mathfrak{M}\} \cdot 1-\sqrt{\left\{\mathfrak{M}_{\mathrm{ax}}-\frac{\mathrm{I}}{2} \mathfrak{M}_{\mathrm{bx}}-\frac{\mathrm{I}}{2} \mathfrak{M}_{\mathrm{cx}}\right\}^{2}+\frac{3}{4}\left\{\mathfrak{M}_{\mathrm{cx}}-\mathfrak{M}_{\mathrm{bx}}\right\}^{2} \cdot \sqrt{\mathfrak{M}_{\mathrm{ax}}{ }^{2}+\mathfrak{M}_{\mathrm{ax}}{ }^{2}}}}{\sqrt{\left\{\mathfrak{M}_{\mathrm{ax}}-\frac{I}{2} \mathfrak{M}_{\mathrm{bx}}-\frac{\mathrm{I}}{2} \mathfrak{M}_{\mathrm{cx}}\right\}^{2}+\frac{3}{4}\left\{\mathfrak{M}_{\mathrm{c} x}-\mathfrak{M}_{\mathrm{bx}}\right\}^{2}} \cdot \sqrt{\mathfrak{M}_{\mathrm{ax}}{ }^{2}+\mathfrak{M}_{\mathrm{a} \mathrm{y}}{ }^{2}}}
\end{aligned}
$$

6. Eine andere Art der Berechnung des Heylandschen Streukoeffizienten. Der soeben aus den Leerlaufsgleichungen ermittelte Wert für $\sigma$ ist wieder noch insofern willkürlich, als auch hier der planimetrische Mittelwert aus den verschiedenen Einzelwerten genommen ist; es ist deshalb zu überlegen, inwieweit er andererseits aus der Gleichung

$$
\sigma=\frac{\mathrm{J}_{0}}{\mathrm{~J}_{\mathrm{k}}-\overline{\mathrm{J}_{0}}}
$$

ermittelt werden kann. $\mathrm{J}$ ist hier der ideelle Kurzschlußstrom.

Diesen ideellen Wert bekommt man bei vollkommen symmetrischen Gebilden aus den exakten Kurzschlußgleichungen, wenn man die Widerstände vernachlässigt.

Anders bei unsymmetrischen Gebilden; hier messen wir schon von vornherein verschiedene Werte des Kurzschlußstromes. Diese Verschiedenheiten werden umso größer, je kleiner der sekundäre Widerstand ist. Beim vorliegenden Motor müssen wir also den Wert $w$ in unseren Gleichungen beibehalten.

Der Punkt für $\mathrm{w}=0$ im Kreisdiagramm kann aber theoretisch auch erreicht werden für $\omega_{g_{2}}= \pm \infty$, d. h. in den praktisch nicht realisierbaren Fällen, daß der Rotor mit unendlich großer Geschwindigkeit angetrieben wird. Für einen Idealmotor mit Sinoidalfeldern ergibt sich bekanntlich auch für beliebig große Tourenzahlen, daß die primären Größen nur von $\omega_{1}$, die sekundären nur von $\omega_{1}-\omega_{2}$ abhängig sind.

Eine vorhandene Unsymmetrie können wir nun dadurch zum Ausdruck bringen, daß wir diegegenseitigen Induktions koeffizienten $\mathfrak{M}$ nicht mehr als einfache Sinusfunktionen auffassen, sondern ihnen eine beliebige höhere Harmonische zuordnen. Da die Kurven für $\mathfrak{M}$ stets symmetrische sind, so ist die betreffende Harmonische von ungerader Ordnung und ohne Phasenverschiebung. Wir schreiben also unter der Annahme, daß zurzeit $t=o$ die Phase a gerade der Phase $\mathrm{x}$ gegenüberliegt,

$$
\begin{aligned}
& \mathfrak{M}_{\mathrm{ax}}=\mathfrak{M} \cdot \cos \omega_{2} t+G \cdot \cos g \omega_{2} t \\
& \mathbb{M}_{\mathrm{bx}}=\mathfrak{M} \cdot \cos \left(\omega_{2} t+\mathrm{I} 20\right)+G \cdot \cos \left(g \omega_{2} t+120\right) \\
& \mathbb{M}_{\mathrm{cx}}=\mathfrak{M} \cdot \cos \left(\omega_{2} t+240\right)+G \cdot \cos \left(g \omega_{2} t+240\right) \\
& \mathbb{M}_{\text {ay }}=\mathfrak{M} \cdot \sin \omega_{2} t+G \cdot \sin g \omega_{2} t
\end{aligned}
$$

Fragen wir jetzt der Einfachheit halber wieder zunächst, welche Spannung primär anzulegen ist, wenn wir einen sinusförmigen Strom J durch den Stator hindurchzwingen wollen bei $\omega_{2}=\infty$, so erhalten wir:

$$
\begin{aligned}
\mathrm{e}_{\mathrm{at}} & \left.=\mathrm{J}\left\{\mathrm{W} \cdot \sin \omega_{1} \mathrm{t}+\omega_{1}\left(\mathrm{~L}+\mathfrak{M}^{t}\right) \cos \omega_{1} \mathrm{t}\right)\right\}+\omega_{1} \frac{\mathfrak{M}^{2} \cdot J}{\mathrm{I}} \cos \omega_{1} t \\
& +\left\{\omega_{1}+(\mathrm{g}-\mathrm{I}) \omega_{2}\right\} \cdot \frac{\mathrm{G} \cdot \mathfrak{M}^{2} \mathrm{~J}}{1} \cos \left\{\left[\omega_{1}+(\mathrm{g}-\mathrm{I}) \omega_{2}\right] \cdot \mathrm{t}\right\},
\end{aligned}
$$

d. h. es muß der beim Idealmotor vorhandenen Klemmenspannung eine $\infty$ große Spannung von $(\mathrm{g}-\mathrm{I}) \cdot \infty$ großer Periodenzahl ïbergelagert werden.

Oder umgekehrt, gehen wir von einer sinusförmigen Netzspannung aus, so sind dem Strom sicherlich Oberwellen übergelagert, deren Größe aber wie bei allen Problemen der 
Ankerrückwirkung von den Daten des Netzes, der Selbstinduktion, der Kapazität und des Widerstandes abhängig ist. Auch auf diesem Wege scheinen wir also nicht zu einem eindeutigen Werte der Streuung zu gelangen. Betrachten wir dagegen den Umstand genauer, daß alle Oberwellen gerade bei hohen Periodenzahlen durch Wirbelströme kompensiert werden, so scheint der Weg viel für sich zu haben, die Grundwelle von $\mathfrak{M}$ zu bestimmen, und allein diese in die Rechnung einzuführen. Wenigstens läßt sich dieser Vorschlag gerade so gut verteidigen wie die Errechnung des planimetrischen Mittelwertes.

Will man beide Werte prinzipiell miteinander vergleichen, so kann man mathematisch etwa folgendermaßen vorgehen:

Man errechnet den Mittelwert des Streukoeffizienten derart, daß man wieder außer der Grundwelle der Kurve für $\mathfrak{M}$ noch eine beliebige höhere Harmonische zuläßt, und zwar geht man der Einfachheit halber aus vom $Z$ weiphasenmotor. Die früher ermittelte Formel für a lautete:

Wir setzen nun

$$
\sigma=\frac{\mathrm{L} \cdot 1-\left(\mathrm{M}_{1}^{2}+\mathrm{M}_{2}^{2}\right)}{\mathrm{M}_{1}^{2}+\mathrm{M}_{2}^{2}}
$$

$$
\begin{aligned}
& \mathrm{M}_{1}=\mathfrak{M} \cdot \sin \alpha+\mathrm{G} \cdot \sin \mathrm{g} \alpha \quad \text { und } \\
& \mathrm{M}_{2}=\mathfrak{M} \cdot \cos \alpha+\mathrm{G} \cdot \cos \mathrm{g} \alpha
\end{aligned}
$$

Hiernach ergibt sich $\sigma$ zu:

$$
\sigma=\frac{\mathrm{L} \cdot 1}{(\mathfrak{M} \cdot \sin \alpha+\mathrm{G} \cdot \sin g \alpha)^{2}+(\mathfrak{M} \cdot \cos \alpha+\mathrm{G} \cdot \cos g \alpha)^{2}}-\mathrm{I}
$$

und der gesuchte Mittelwert $\sigma_{\mathrm{m}}$ ist:

$$
\sigma_{\mathrm{m}}=\frac{2}{\pi} \int_{0}^{\frac{\pi}{2}} \frac{\mathrm{L} \cdot 1 \cdot \mathrm{d} \alpha}{(\mathfrak{M} \cdot \sin \alpha+\mathrm{G} \cdot \sin \cdot \mathrm{g} \alpha)^{2}+(\mathfrak{M} \cos \alpha+\mathrm{G} \cos \mathrm{g} \alpha)^{2}}-\mathrm{I} .
$$

Das Integral

$$
\int \frac{\mathrm{d} \alpha}{(\mathfrak{M} \sin \alpha+\mathrm{G} \cdot \sin g \alpha)^{2}+(\mathfrak{M} \cos \alpha+G \cos g \alpha)^{2}}
$$

kann durch Entwicklung des Nenners in

$$
\int \frac{d \alpha}{\mathfrak{M}^{2}+G^{2}+2 \mathfrak{M G} \cos [(g-I) \alpha]}
$$

und durch die Substitution

$$
(\mathrm{g}-\mathrm{I}) \alpha=\mathrm{x}
$$

in

$$
\frac{I}{g-I} \int \frac{d x}{M^{2}+G^{2}+2 M G \cos x}
$$

übergeführt werden.

Die Lösung dieses Integrales ist, da im vorliegenden Falle

die folgende

$$
\mathfrak{M R}^{4}+\mathrm{G}^{4}>2 \mathfrak{M}^{2} \mathrm{G}^{2},
$$

$$
\begin{aligned}
& \frac{I}{g-I} \int \frac{d x}{M^{2}+G^{2}+2 M G \cos x}= \\
& \frac{I}{g-I} \cdot \frac{2}{\sqrt{M^{4}+G^{4}-2 M^{2} G^{2}}} \cdot \operatorname{arctg}\left\{\sqrt{\frac{M^{2}+G^{2}-2 \mathfrak{M G}}{M^{2}+G^{2}+2 \mathfrak{M} G}} \cdot \operatorname{tg} \frac{x}{2}\right\} .
\end{aligned}
$$


Für den Mittelwert $\sigma_{\mathrm{m}}$ erhalten wir demnach, da die obere Grenze des Integrales $(\mathrm{g}-\mathrm{I}) \frac{\pi}{2}$ ist;

$$
\sigma_{m}=\frac{4 \cdot \operatorname{arctg} \frac{M-G}{M+G} \cdot \operatorname{tg}\left\{(g-I) \frac{\pi}{4}\right\}}{\pi \cdot(g-I) \cdot\left(M^{2}-G^{2}\right)} \cdot L \cdot 1-I .
$$

Diese Formel gibt also direkt die Abweichung für den genannten Fall gegenüber dem Streukoeffizienten

$$
\sigma^{\prime}=\frac{\mathrm{L} \cdot \mathrm{I}}{\mathfrak{M}^{2}}-\mathrm{I}
$$

der ohne Berücksichtigung der Oberwelle $(G, g)$ errechnet wird.

Die Formel geht, wie zu verlangen, unter der Voraussetzung in den Wert $\sigma^{\prime}$ über, daß G vernachlässigbar klein ist gegen $\mathfrak{M}$.

Bei den den Verlauf von $\mathfrak{M}$ darstellenden gebrochenen Linienzügen haben wir eine so große Annäherung an die Sinuslinie, daß wir hier ohne Bedenken uns auf die Betrachtung der Grundwelle beschränken können. Man überzeugt sich hiervon leicht durch eine Zerlegung von $\mathfrak{M}$ in seine Harmonischen; da mit zunehmenden Nutenzahlen die Differenz zwischen der wahren Kurve und einer Sinuskurve kleiner wird, so sei hier der krasseste Fall, der Zweiphasenmotor mit 2 Nuten primär und 3 Nuten sekundär, bezogen auf einen Pol und eine Phase, angeführt.

Mit einem harmonischen Analysator ${ }^{1}$ ) ist die Gleichung für M II $2 / 3$ gefunden:

$$
\mathfrak{M}=0,9833 \sin \alpha \ldots 0,0133 \sin 3 \alpha+0,0033 \sin 5 \alpha .
$$

Hier beträgt die Amplitude der dritten Oberwelle den etwa 74 ten Teil der Amplitude der Grundwelle, das sind etwa I,33\%. Mit Rücksicht auf die für die Bedürfnisse der Praxis erforderliche Genauigkeit ist die Vernachlässigung der Oberwellen also durchaus statthaft. Wir bekommen auf diese Art den Mittelwert von $\sigma \mathrm{zu}$

$$
\sigma_{\mathrm{m}}^{\prime \prime}=0, \mathrm{I} 3 \mathrm{I} 8 \text {. }
$$

Die Abweichungen ${ }^{2}$ ) von den früher ermittelten Werten für $\sigma_{\mathrm{m}}$ sind

$-0,53 \%$, bez. a. d. planim. Mittelwert,

+5, r \%, bez. a. d. einfachen Mittelwert.

7. Beispiel. I. Errechnung aus der Blondelschen Definition und Bestimmung des planimetrischen Mittelwertes. Wir behandeln einen Motor mit primär 3 Nuten pro Pol und Phase und sekundär 4 Nuten pro Pol und Phase, das Verhältnis der Nutenzahlen ist also $9 / 8$.

Wie im Teil I betrachten wir wieder Wechselfelder, errechnen die charakteristischen Punkte von $\mathfrak{M}$ und gelangen so zu der Figur 9:

Für eine Anzahl von Punkten bestimmen wir die Werte von $\sigma$, siehe folgende Figur, und bilden den planimetrischen Mittelwert $\sigma_{\mathrm{m}}$ :

$$
\sigma_{\mathrm{m}}=0,0420 .
$$

2. Vergleich mit der Berechnung unter Zugrundelegung allein der Grundwelle von $\mathfrak{M}$. Wir greifen eine Kurve heraus, beispielsweise $\mathfrak{M}_{\text {ax }}$ und zeichnen für den Bereich o bis $90_{0}$ die Kurve $\mathfrak{M}_{\mathrm{ax}} \cdot \sin \alpha$. Diese Kurve planimetrieren wir und finden

1) Nach Dr. Ing. O. Mader; siebe ETZ. I909, Heft 36.

$\left.{ }^{2}\right)$ Bei der Abschätzung der Größe der Fehler ist zu berücksichtigen, daß in allen Mittelwerten Zeichen- und Ablesungsfehler (Planimeter, Analysator) enthalten sind. 
in dem doppelten Mittelwert dieser Kurve die Amplitude der Grundwelle von $\Re$. Unter Zugrundelegung dieser Grundwelle ergibt sich ein $\sigma_{\mathrm{m}}^{\prime}$ von

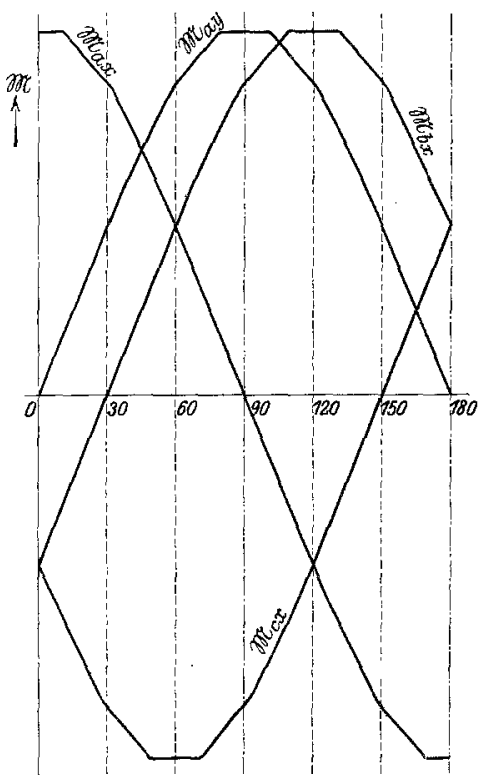

Fig. 9.

$$
\sigma_{\mathrm{m}}^{\prime}=0,0406 .
$$

Die Abweichung beträgt 3,23\%, bezogen auf den planimetrischen Mittelwert.

In gleicher Weise sind zur Kontrolle weitere Beispiele durchgeführt; stets lagen die $\mathrm{Ab}$ weichungen vom planimetrischen Mittelwert innerhalb der Grenzen der Genauigkeit beim Zeichnen. Der bequemeren Rechnung wegen wird man sich daher gern der zuletzt angegebenen Methode - der Berücksichtigung allein der Grundwelle -- bedienen.

Die Kurven für den Koeffizienten $\sigma$ der doppelt verketteten Streuung sind jetzt nicht mehr symmetrisch zur Ordinate für $\frac{\pi}{4}$, vielmehr nur zur Ordinate für $\frac{\pi}{2}$; die Periode ist die doppelte derjenigen der Kurven für $\sigma$ bei $Z$ weiphasenmotoren, wie es Figur ro deutlich erkennen läßt.

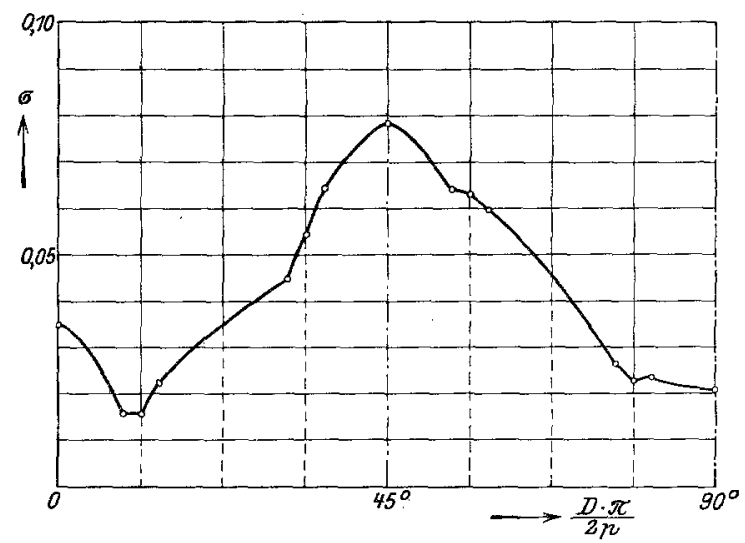

Fig. Io.

8. Diskussion der Ergebnisse. Die Vorzüge und Nachteile der einzelnen Motoren bezüglich ihrer Streuungsverhältnisse unter Berücksichtigung der mechanischen Herstellung werden am deutlichsten hervortreten, wenn wir im folgenden je einen Drehstrom-, Zweiphasenmotor und einen aus beiden Systemen kombinierten Motor einander gegenüberstellen.

Die Rechnungsergebnisse des ersten Teiles lassen klar erkennen, daß, um die Größe des Streukoeffizienten klein zu halten, die geringste Differenz zwischen primärer und sekundärer Nutenzahl anzustreben ist. Gleiche Nutenzahlen müssen für die praktischen Bedürfnisse ausgeschaltet werden, da so gebaute Motoren kein Anlaufsmoment zu entwickeln vermögen.

Beim Drehstrommotor ist die kleinste Differenz der Nutenzahlen pro Pol gleich 3, beim Zweiphasenmotor gleich 2 und beim Zweiphasenrotor im Dreiphasenfeld gleich $\mathrm{I}$. Hier also haben primäre und sekundäre Gesamtnutenzahl pro Pol keinen gemeinsamen 
Teiler, die Anlaufsverhältnisse sind demnach sehr günstig. Wir wählen einen Motor dieser Bauart, und zwar den im vorigen Beispiel behandelten. Die Summe seiner Nutenzahl en, auf einen Pol bezogen, ist

$$
9+8=\text { I7 }
$$

Weder mit Dreiphasen- noch mit Zweiphasenwicklung läßt sich diese Zahl erreichen. Die nächsten Näherungswerte sind für Drehstrommotore $2 / 3$ und $3 / 3$ Motore mit:

$$
\begin{aligned}
& 3 \times 2+3 \times 3=\text { I5 Nuten. } \\
& 3 \times 3+3 \times 3=\text { I } 8,
\end{aligned}
$$

Der Motor mit I5 Nuten läuft mäßig an, der mit I 8 Nuten überhaupt nicht.

Die Mittelwerte des Streukoeffizienten sind:

$$
\begin{aligned}
& \sigma_{\mathrm{m}}=0,042 \text { für } N_{1}+N_{2}=\mathrm{I}_{5}, \\
& \sigma_{\mathrm{m}}=0,03 \mathrm{I} \text { für } N_{1}+N_{2}=\mathrm{I}^{1} \text { ). }
\end{aligned}
$$

Als Zweiphasenmotoren kommen in Betracht $3 / 5$ und $4 / 5$ mit:

$$
\begin{aligned}
& N_{1}+N_{2}=I 6 \text { Nuten, } \\
& N_{1}+N_{2}=I 8
\end{aligned}
$$

Beide Motoren laufen ziemlich gut an; für $\sigma_{m}$ ergibt $\left.\operatorname{sich}^{2}\right)$ :

$$
\begin{aligned}
& \sigma_{\mathrm{m}}=0,0736 \text { für } \mathrm{N}_{1}+\mathrm{N}_{2}=\mathrm{I} 6, \\
& \sigma_{\mathrm{m}}=0,0604 \text { für } \mathrm{N}_{1}+\mathrm{N}_{2}=\mathrm{I} 8 .
\end{aligned}
$$

Der aus beiden kombinierte Motor mit $\mathrm{N}_{1}+\mathrm{N}_{2}=\Upsilon 7$ weist als mittleren Streukoeffizienten auf:

$$
\sigma_{\mathrm{m}}=0,0406 .
$$

Die Wahl ist nun zu treffen zwischen:

$$
\begin{array}{r}
\text { Drehstrommotor; } N_{1}+N_{2}=15 ; \sigma_{\mathrm{m}}=0,042 \\
\text { Zweiphasenmotor; } N_{1}+N_{2}=18 ; \sigma_{\mathrm{m}}=0,0604 \\
\text { Zwei- und Dreiph.-Motor; } N_{1}+N_{2}=17 ; \sigma_{\mathrm{m}}=0,0406
\end{array}
$$

Der Drehstrommotor hat die kleinste Teilung, wird in seinen Dimensionen am kleinsten bleiben und verlangt den geringsten Aufwand an Material und Arbeit zur Herstellung seines Eisenteiles mit zugehöriger Wicklung, dagegen sind seine Anlaufsverhältnisse ungünstig.

Beim Motor mit 2--und 3-Phasen-Wicklung hat man pro Pol zwei Nuten mehr zu stanzen und mit Wicklungsmaterial zu füllen, erreicht dafür ein sehr gutes Anlaufen und noch geringere Streuung als beim eben erwähnten Drehstrommotor.

Man wird sich daher, besonders wenn es sich um große, Iangsam laufende Maschinen handelt, jedenfalls für die Ausführung als $Z$ weiphasenmotor im Dreiphasenfeld entscheiden.

Der $Z$ weiphasenmotor kann im vorliegenden Fall mit keinem der anderen Typen konkurrieren.

1) Entnommen der Arbeit von Rogowski, ETz. rgro, Heft 5 I.

2) Vgl. Tabelle im I. Teil. 
Um elektrische Maschinen bezüglich ihrer Verhältnisse der doppelt verketteten Streuung in größerer Allgemeinheit vergleichen zu können, müßte man noch andere Wicklungsarten als die hier behandelten untersuchen.

Jedenfalls aber geht aus dem Vorstehenden schon hervor, daß, obwohl der Drehstrommotor im allgemeinen die geringste Streuung zeigt, aus mechanischen Gründen und wegen der Anlaufsverhältnisse dem Dreiphasenstator mit Zweiphasenrotor in gewissen Fällen der Vorzug gegeben werden kann.

\section{Berichtigung.}

In der im 6. Heft des Archivs erschienenen Arbeit von W. Petersen ,Wanderwellen als Uberspannungserreger" soll es heißen:

S. 243, Abschnitt 7, 2. Absatz, I. Zeile: . . Gesamtlänge 2 l;

S. 244, 3. Absatz: . . gleiche Iänge wie der Abschnitt A oder B, so herrscht . . 